\title{
RESEARCH
}

Open Access

\section{The circACTN4 interacts with FUBP1 to promote tumorigenesis and progression of breast cancer by regulating the expression of proto-oncogene MYC}

Xiaosong Wang ${ }^{1}$, Lei Xing ${ }^{2}$, Rui Yang ${ }^{1}$, Hang Chen ${ }^{1}$, Min Wang ${ }^{1}$, Rong Jiang ${ }^{3}$, Luyu Zhang ${ }^{4}$ and Junxia Chen ${ }^{1 *}$ (D)

\begin{abstract}
Background: Recent studies have revealed that circular RNAs (circRNAs) play significant roles in the occurrence and development of many kinds of cancers including breast cancer (BC). However, the potential functions of most circRNAs and the molecular mechanisms underlying progression of $B C$ remain elusive.

Method: Here, Circular RNA microarray was executed in 4 pairs of breast cancer tissues and para-cancer tissues. The expression and prognostic significance of circACTN4 in BC cells and tissues were determined by qRT-PCR and in situ hybridization. Gain-and loss-of-function experiments were implemented to observe the impacts of circACTN4 on the growth, invasion, and metastasis of BC cells in vitro and in vivo. Mechanistically, chromatin immunoprecipitation, luciferase reporter, RNA pulldown, mass spectrum, RNA immunoprecipitation, fluorescence in situ hybridization and co-immunoprecipitation assays were executed.

Results: CircACTN4 was significantly upregulated in breast cancer tissues and cells, its expression was correlated with clinical stage and poor prognosis of patients with BC. Ectopic expression of circACTN4 strikingly facilitated the growth, invasion, and metastasis of breast cancer cells in vitro and in vivo. Whereas knockdown of circACTN4 revealed opposite roles. CircACTN4 was mainly distributed in the nucleus. Further mechanistic research proved that circACTN4 could competitively bind to far upstream element binding protein 1 (FUBP1) to prevent the combination between FUBP1 and FIR, thereby activating MYC transcription and facilitating tumor progression of breast cancer. Furthermore, we found that upstream transcription factor 2 (USF2) might promote the biogenesis of circACTN4.

Conclusion: Our findings uncover a pivotal mechanism that circACTN4 mediated by USF2 might interact with FUBP1 to promote the occurrence and development of breast cancer via enhancing the expression of MYC. CircACTN4 could be a novel potential target for diagnosis and treatment of breast cancer.
\end{abstract}

Keywords: circactn4, FUBP1, The RNA-binding protein, MYC, Breast cancer

\footnotetext{
*Correspondence: chjunxia@126.com

'Department of Cell Biology and Genetics, Chongqing Medical University, \#1 Yixueyuan Road, Chongqing 400016, China

Full list of author information is available at the end of the article
}

(c) The Author(s). 2021 Open Access This article is licensed under a Creative Commons Attribution 4.0 International License, which permits use, sharing, adaptation, distribution and reproduction in any medium or format, as long as you give appropriate credit to the original author(s) and the source, provide a link to the Creative Commons licence, and indicate if changes were made. The images or other third party material in this article are included in the article's Creative Commons licence, unless indicated otherwise in a credit line to the material. If material is not included in the article's Creative Commons licence and your intended use is not permitted by statutory regulation or exceeds the permitted use, you will need to obtain permission directly from the copyright holder. To view a copy of this licence, visit http://creativecommons.org/licenses/by/4.0/ The Creative Commons Public Domain Dedication waiver (http://creativecommons.org/publicdomain/zero/1.0/) applies to the data made available in this article, unless otherwise stated in a credit line to the data. 


\section{Background}

Breast cancer is the malignant tumor with the highest incidence among women in the world. Globally, about 2.1 million cases of breast cancer were newly diagnosed in 2018, accounting for about $25 \%$ of all female malignant tumor cases. Although the early detection and effective systematic treatment of breast cancer have improved, it remains the leading cause of cancer death in women all over the world [1]. Thus, there is an urgent need to find new therapeutic targets and strategies for BC.

Circular RNAs (circRNAs) are a new class of RNAs that have been widely found in various species through high-throughput sequencing in recent years. CircRNAs are covalently closed, single-stranded transcripts generated by pre-mRNA back-splicing without 5 ' caps and 3' tails. Due to the covalent loop structure, circRNAs are resistant to RNA exonucleases and are more stable than linear RNA [2]. CircRNAs reveal disease-specific and development stage-specific features in different pathological circumstances, which suggests that circRNAs could be served as novel potential biomarkers for diagnosis and treatment [3]. Recent researches have demonstrated that circRNAs have important potential roles in regulating gene expression. CircRNAs exert their functions, including as miRNA sponges, RNA-binding protein scaffolds, intermediates in RNA alternative splicing (AS), protein translation templates and transcription regulators [4]. However, the interaction of circRNAs and proteins were less studied in human cancers.

Accumulating evidence has indicated that the dysregulated circRNAs are involved in the development of various cancers. The most commonly reported mechanism of circRNAs can function as miRNA sponges to relieve the suppression for miRNA targeted gene expression [57]. In breast cancer, circRNAs, such as circRNA 0006528, circRAD18, circHMCU and circRNF20, have been revealed powerful potential in regulating cell proliferation, migration, metabolism and tumor progression, and their expressions are correlated with high TNM stage and prognosis [8-11]. Recently, researches have reported that circRNAs could interact with RNA binding proteins (RBPs) to exert biological functions [12, 13]. For example, circular RNA CDR1as could directly combine with the p53 DBD domain, thus disrupting the p53/ MDM2 complex formation to suppress gliomagenesis [14]. Circ0005276 increases the proliferation and mobility of prostate cancer cells through binding with FUS to transcriptionally activate XIAP [15]. The RNA-binding protein RBM3 regulates the generation of circular RNA SCD-circRNA 2 to facilitate cell proliferation in hepatocellular carcinoma [16]. However, the interaction and relationship between circRNAs and proteins in breast cancer remain largely unknown.
The human far upstream element (FUSE) binding protein 1 (FUBP1), a multifunctional DNA- and RNAbinding protein, is implicated in transcription, translation, and RNA splicing cellular processes. It is well known that FUBP1 can bind to FUSE upstream of cMYC promoter to recruit and activate transcription factor TFIIH, thus promoting the transcription of c-myc gene. FUBP1 also might bind with the FBP interacting repressor (FIR) to form inhibitory complexes with FUSE and TFIIH, and inhibit c-MYC transcription. The interplay of FUSE, FUBP1 and FIR in this molecular machinery can fine-tune c-myc transcription in a real-time manner [17]. FUBP1 overexpression was found in many cancers and led to a dysregulation of targets including MYC oncogene [18]. The study have shown that the expressions of FUBP1 and its target MYC in breast cancer tissues were higher than those in adjacent normal tissues [19]. It has been reported that lncRNA SNHG1 could directly interact with FUBP1 central domain and resist the binding between FIR and FUBP1, thereby modulating the oncogene MYC transcription and promoting cancer development [20]. To date, the association of between circRNAs and FUBP1 has not been explored.

In this study, we performed circular RNA microarray and displayed the circRNA expression profile in breast cancer tissues. Then we focused on a novel circRNA circACTN4 (hsa_circ_0050900) from ACTN4 gene and investigated its biological functions as well as the underlying molecular mechanisms in the breast cancer development. The results showed that circACTN4 was highly expressed in $\mathrm{BC}$ tissues and cells, which was positively correlated with advanced tumor stage and poor prognosis. Moreover, we demonstrated that USF2 could facilitate the generation of circACTN4. Our functional investigation displayed that circACTN4 could enhance $\mathrm{BC}$ cell growth and metastasis. Further mechanistic research revealed that circACTN4 could competitively bind to FUBP1 and block the binding of FUBP1 with FIR, thus promoting the transcription of MYC and development of breast cancer. Our results suggest that circACTN4 could be a new therapeutic target and promising prognostic biomarker for BC.

\section{Materials and methods}

\section{Clinical BC tissues and cell lines}

Cancer and para-cancer tissues of 80 breast cancer patients who did not receive preoperative chemotherapy or radiotherapy were obtained at the First Affiliated Hospital of Chongqing Medical University (Chongqing, China). Fresh tissues were saved in liquid nitrogen or stored at $-80^{\circ} \mathrm{C}$ until RNA extraction. All procedures were implemented according to the guidelines of the Declaration of Helsinki. All patients provided written informed consent. Human normal breast epithelial MCF- 
10A cells and breast cancer MCF-7 and SK-BR-3 cells as well as $293 \mathrm{~T}$ cells were preserved in our laboratory, breast cancer ZR-75-1, BT-474 and T-47D cells were bought from American Type Culture Collection (ATCC, Manassas, VA, USA). MCF-10A cells were cultivated in MEBM BulletKit medium (Lonza, Basel, Switzerland), MCF-7, T-47D and $293 \mathrm{~T}$ cells were maintained in DMEM (Gibco, Carlsbad, CA, USA) with 10\% fetal bovine serum (FBS), SK-BR-3, ZR-75-1 and BT-474 were fostered in RPMI-1640 (Gibco, Carlsbad, CA, USA) with $10 \%$ or $20 \%$ FBS. All media were supplemented with $1 \%$ penicillin/streptomycin. All cells were placed in a 5\% $\mathrm{CO}_{2}$ incubator at $37^{\circ} \mathrm{C}$.

\section{Plasmid construction, RNAi and stable transfection}

circACTN4 overexpression vector were designed and the human full-length circACTN4 was inserted into vector pLC5-ciR by Geenseed (Guangzhou, China), the mock vector without circACTN4 sequence was served as a control. To knock down circACTN4, siRNAs (small interfering RNAs) targeting back splice junction of circACTN4 (si-circ\#1, si-circ\#2) and siRNA-NC were synthesized by Geenseed (Guangzhou, China). Human circACTN4 gene was inserted into the lentivirus vector CV146 (Ubi-MCS-SV40-firefly_Luciferase-IRES-Puromycin). The si-circ\#2 was subcloned into the lentivirus vector GV344 (hU6- MCS- Ubiquitin- firefly_LuciferaseIRES- puromycin) by Genechem (Shanghai, China). Overexpression vectors of human USF2, FUBP1, FIR and ACTN4 gene were synthesized and ligated into GV146 (CMV-MCS-IRES-EGFP-SV40-Neomycin) by Genechem (Shanghai, China). Short-hairpin RNAs (shRNAs) directed against USF2, FUBP1, FIR and ACTN4 gene were constructed in GV102 (hU6-MCSCMV-GFP-SV40-Neomycin) vectors (Genechem Co., Ltd., Shanghai, China). The lentiviral vector was transiently transfected into HEK293T cells. The viral supernatant was collected for animal experiments. Stable cell lines were screened with neomycin or puromycin. All transfections were implemented using Lipofectamine 3000 (Invitrogen, Carlsbad, CA, USA) according to manufacturer's instructions. The sequences of shRNAs and siRNAs used were displayed in Additional file 1: Table S2.

\section{Animal experiments}

Four-week-old female BALB/c nude mice were injected subcutaneously with $10^{7}$ MCF-7 cells infected with various lentiviruses. All processes were executed according to NIH Guidelines for the Care and Use of Laboratory Animals and approved by Chongqing Medical University Animal Care and Use Committee. The width and length of the tumor were measured once every week, and tumor volume was calculated based on the formula (length $\times$ width $^{2} / 2$ ). Mice were sacrificed and tumors were harvested and weighed after 4 weeks. The tumors and lungs were obtained for the next study. The mice were administered subcutaneously with cells infected with the lentiviral vector overexpressing circACTN4 or the mock lentiviral respectively ( $n=10$ per group) for survival analysis, and were observed for 2 months as a cutoff. After 2 months, the mice still alive were regarded to be censored, the mice were executed and the livers were excised for pathological analysis. MCF-7 cells were stably infected with luciferase-labeled mock, overexpressed circACTN4, small interfering control (si-NC), and si-circ\#2 lentivirus. $4 \times 10^{6}$ cells were injected into female BALB/c nude mice tail vein. After 4 weeks, 150 $\mathrm{mg} / \mathrm{kg}$ D-Luciferin (Sciencelight Biology Science \& Technology Co., Ltd. Shanghai, China) was injected by intraperitoneal injection, After $15 \mathrm{~min}$, the mice were imaged for luciferase activity using the Nightowl LB 983 in vivo imaging system (Berthold, Germany).

\section{Microarray analysis}

Total RNA of 4 pairs of non-triple negative breast cancer and para-cancer tissues was extracted with TRIzol reagent (Takara, Dalian, China) and quantified by the NanoDrop ND-1000 (Thermo Fisher Scientific, USA), then digested with RNase $\mathrm{R}$ to eliminate linear RNAs and enrich circular RNAs. The sample preparing and microarray hybriding were executed following the Arraystar's standard protocols by KangChen Bio-tech (Shanghai, China). Microarray analysis of circRNA was implemented by Arraystar Human circRNA Array V2. preparing sample.

\section{Nuclear-cytoplasmic fractionation, RT-PCR and qRT-PCR assays}

Total RNA from tissues and cells was extracted with TRIzol reagent (Takara, Dalian, China). Nuclear and cytoplasmic RNA in MCF-7 cells was isolated using the PARIS $^{\text {ma }}$ Kit (Life Technologies, Austin, Texas, USA) according to the manufacturer's protocols. RNase R (Epicentre Biotechnologies, Madison, WI, USA) digestions with $4 \mathrm{U} / \mu \mathrm{g}$ were performed for $0,10,20,30$ and $40 \mathrm{~min}$ at $37^{\circ} \mathrm{C}$. One hundred nanograms per milliliter actinomycin D (Cell Signaling Technology, Beverly, MA, USA) was used to treat total RNA from BC cells against new RNA synthesis for 12 and $24 \mathrm{~h}$. The semi-quantitative PCR amplification cycle was 39. Reverse transcription was conducted by PrimeScript RT Reagent Kit (Takara, Dalian, China), and quantitative real-time PCR (qRTPCR) analysis was executed with the TB Green Premix Ex Taq (Takara, Dalian, China). The $2^{-\Delta \Delta \mathrm{Ct}}$ method was used to analyze the relative expression levels. The primer sequences were listed in Additional file 1: Table S1. 


\section{Fluorescence in Situ Hybridization (FISH) and} Immunofluorescence (IF)

Cy3-labeled probe for circACTN4 was synthesized (Geneseed, Guangzhou, China). BC cells were cultured on coverslips, incubated with antibodies specific for FUBP1 (Thermo fisher scientific, USA) at $4{ }^{\circ} \mathrm{C}$ overnight, and treated with FITC-labeled goat anti-rabbit IgG (Bosterbio, Wuhan, China; 1:1000 dilution) at $37^{\circ} \mathrm{C}$ for $2 \mathrm{~h}$, and then incubated with FISH probe in hybridization buffer (Geneseed, Guangzhou, China) at $37^{\circ} \mathrm{C}$ for $16 \mathrm{~h}$. the cell nuclei were stained by DAPI(4'6- diamidino- 2phenylindole). These images were taken with Olympus BX51 fluorescence microscope (Tokyo, Japan). The probe sequences were displayed in Additional file 1: Table S3

\section{Tissue Microarray (TMA) and In Situ Hybridization (ISH)}

TMA was produced from 240 paraffin-embedded samples by Outdo Biotech (Shanghai, China). ISH was used to detect the expression of circACTN4 in BC tissues. First, the TMA was dewaxed in xylene and rehydrated with 100, 95, 85 and 75\% alcohol, and then the tissues were hybridized with specific digoxin-labeled circACTN4 probe (Geneseed, Guangzhou, China). The specimens were incubated with AP-conjugated antidigoxin antibody (Roche, Basel, Switzerland) at $4{ }^{\circ} \mathrm{C}$ overnight, than stained with NBT/BCIP (Roche, Basel, Switzerland) and photographed. The expressions of circACTN4 were quantified and analyzed. The positive staining intensity ( 0 , negative; 1 , weak; 2 , moderate; 3 , strong) was multiplied by the percentage of positive staining cells $(0,<10 \%=0 ; 1,10-25 \% ; 2,26-50 \% ; 3,51-$ $75 \% ; 4,>75 \%)$ to calculate the score for ISH staining. The ISH score $\leq 8$ defined low expression, while $>8$ indicated high expression. The probe sequence was displayed in Additional file 1: Table S3.

\section{Dual luciferase reporter assay}

The human ACTN4 promoter luciferase reporter gene vector was constructed, the full-length promoter of ACTN4 carrying mutant or wild type was respectively cloned into pGL3-basic vectors (GeneCreate, Wuhan, China), and co-transfected with USF2 overexpression vector or mock vector into $293 \mathrm{~T}$ cells. The cells were harvested after $48 \mathrm{~h}$, the Dual Luciferase Reporter Assay System Kit (Promega, Madison, WI, USA) was used to detect the activities of firefly and Renilla luciferase.

\section{Cell proliferation, wound healing, migration and invasion} assays

CCK-8, Edu, colony formation, wound healing, transwell migration and invasion assays were carried out as previously reported [21].

\section{Cell cycle and apoptosis analysis}

Forty-eight hours after transfection, cells were resuspended and fixed with ice cold $70 \%$ ethanol overnight at $4{ }^{\circ} \mathrm{Cfor}$ cell cycle analysis, and stained by PI (propidium iodide), than tested using the flow cytometry (Becon Dickinson FACS Calibur, NY, USA). The cells were stained with Annexin V-FITC and PI to detect the cell apoptosis rate with flow cytometry. Hoechst 33342 (Beyotime, Shanghai, China) staining was also utilized to detect cell apoptosis following the manufacturer's instructions and photographed with the fluorescent microscope (Leica, Wetzlar, Germany).

\section{Chromatin immunoprecipitation (ChIP) assay}

ChIP assay was undertaken using EZ ChIP Chromatin Immunoprecipitation Kit (Millipore, Billerica, MD, USA) following the manufacturer's protocols. Briefly, $2 \times 10^{7}$ cells were used for each individual reaction. The MCF-7 cells were fixed by $1 \%$ formaldehyde and lysed. The genome was sonicated $200 \sim 1000$ bp DNA fragments. Ten microgram antibodies specific for USF2 (Abcam, Burlingame, CA, USA), FUBP1 (Thermo Fisher Scientific, USA) and FIR (Cell Signaling Technology, Beverly, MA, USA) were added into the cell lysate for incubation at $4{ }^{\circ} \mathrm{C}$ overnight, than incubated with Protein A Agarose/ Salmon Sperm DNA beads (50\% Slurry). The input DNA and immunoprecipitated DNA was purified and detected by qPCR using the primer sequences (Additional file 1: Table S1).

\section{RNA pull down assay and mass spectrometry}

The interaction between circACTN4 and RNA-bindingprotein was detected by Pierce Magnetic RNA-Protein Pull-Down Kit (Thermo Fisher Scientific, USA) according to the manufacturer's protocols. Biotin-labeled probes targeting junction site of circACTN4 were synthesized by RiboBio (Guangzhou, China) and oligo probe was used as a control. Linear circACTN4 was transcribed with Biotin RNA Labeling Mix (Roche) and T7 RNA polymerase (Thermo Fisher Scientific, USA), circularized using T4 RNA ligase I and digested by RNase R. The cells were lysed and incubated with biotin-labeled circACTN4 probe. Afterwards, cell lysates were subjected to streptavidin agarose magnetic beads at normal temperature. Finally, interacting proteins were identified by mass spectrometry and Western blot (Life Sciences Institute of Chonqing Medical University, Chongqing, China). The sequences of the probe were shown in Additional file 1: Table S3.

\section{RNA immunoprecipitation (RIP) assay}

The interaction between RBP FUBP1 and circACTN4 was detected by RNA immunoprecipitation kit (Geneseed, Guangzhou, China) according to the 
manufacturer's instruction. Briefly, $2 \times 10^{7}$ cells were harvested and lysed by RIP lysis buffer, then incubated with magnetic beads conjugated with antibody against IgG (Cell Signaling Technology, Beverly, MA, USA) or FUBP1 (Thermo Fisher Scientific, USA). The coprecipitated RNAs were examined using qRT-PCR or RT-PCR with specific primers. The primer sequences were listed in Additional file 1: Table S1.

\section{Co-immunoprecipitation (co-IP) assay}

MCF-7 cells were transfected with overexpressed circACTN4 plasmids and si-circ\#2 by lipofectamine 2000 (Thermo Fisher Scientific, USA). Co-IP assay was carried out using a Co- Immunoprecipitation Kit (Millipore, Billerica, MD, USA) following the manufacturer's protocols. In brief, cells were harvested and lysed, then incubated with $10 \mu \mathrm{g}$ IgG or specific for FUBP1 antibody (Thermo Fisher Scientific, USA) coated beads and rotated at $4{ }^{\circ} \mathrm{C}$ overnight. Bead-bound proteins were released and analyzed by Western blot.

\section{Immunohistochemistry (IHC)}

For IHC assay, tissues were fixed with $4 \%$ paraformaldehyde and embedded in paraffin, then cutted into slices. Primary antibodies against MYC (1:8000, Proteintech, USA), CDK4 (1:8000, Proteintech, USA), CCND2 (1:200, Cell Signaling Technology, Beverly, MA, USA) and Ki67 (1:8000, Proteintech,USA) were used. HRP-labeled streptavidin solution was added into the samples for 15 min after both primary and secondary antibody incubations. The immunocomplex was visualized with DAB, and the nucleus were counterstained with haematoxylin. Pictures were taken with a microscope (Leica, Wetzlar, Germany).

\section{Western blotting}

Tissue and cellular proteins were extracted with radioimmunoprecipitation assay buffer (Beyotime, Jiangsu, China) mixed with phenyl-methylsulfonyl fluoride (PMSF), and quantified with BCA protein assay kit (Beyotime, Jiangsu, China). Equal amount of proteins were loaded and analyzed by 10\% SDS-PAGE gels. The isolated proteins were transferred to PVDF membranes (Bio-Rad, CA, USA). After blocking with 5\% fat-free milk, the membranes were incubated with primary antibodies against MYC (1:1000), FUBP1 (1:1000), FIR (1: 1000), nm23-H1(1:1000), MMP2 (1:1000), MMP9 (1: 1000), CDK4 (1:1000), CCND2 (1:1000), CCND1 (1: 1000), CCNE1 (1:1000), Bcl-2 (1:1000), Bax (1:1000) and cleaved Caspase-3 (1:1000) (Cell Signaling Technology, Beverly, MA, USA) overnight at $4{ }^{\circ} \mathrm{C}$. The membranes were washed by TBST, and then incubated with goat anti-rabbit secondary antibodies (Thermo Fisher
Scientific, USA) for $2 \mathrm{~h}$. Finally, the bands were illuminated with Pierce ECL (Thermo Fisher Scientific, USA).

\section{Statistical analysis}

Statistical analyses were conducted by GraphPad Prism 7.0 (GraphPad Software Inc., CA, USA) and SPSS 21.0 (IBM, SPSS, Chicago, IL, USA). Data are expressed as the mean \pm S.D. (Standard Deviation). The comparisons of two groups were computed using two-tailed Student's $t$ test, ANOVA or $x^{2}$ test. Correlations were analyzed by Pearson's correlation test. Univariate and multivariate Cox proportional hazards regression models were used to identify factors associated with survival. The survival analysis was conducted with the Kaplan-Meier plots and the log-rank test. Differences were statistically significant at $P<0.05$.

\section{Results \\ CircACTN4 is identified and USF2 promotes expression of circACTN4 in BC}

To investigate the function of circRNAs in the development of $\mathrm{BC}$, the circRNA expression signatures in $\mathrm{BC}$ tissues were probed utilizing microarray analysis of 4 pairs of $\mathrm{BC}$ tissues and para-cancer tissues. When we use fold-change $\geq 2$ and $P$-value $<0.05$ as the cut-off criteria, we found 85 significantly differentially expressed circRNAs, including 63 up-regulated and 22 downregulated circRNAs. The heatmap shows 14 upregulated circRNAs, among which circACTN4 (hsa_circ_0050900) was the most dysregulated one, and its expression in breast cancer tissues was 10 fold higher than that in adjacent tissues (Fig. 1a). According to circBase (http:// www.circbase.org/), we found that circACTN4 was from the ACTN4 gene located on human chromosome (chr) 19 , and it was generated from exon 2 to exon 7 of ACTN4 (total $571 \mathrm{bp}$ ) by back -splicing, the back splicing junction was verified by Sanger sequencing (Fig. 1b). To verify that circACTN4 is produced by the head to tail splicing rather than trans splicing or genome rearrangement, the convergent and divergent primers were used to amplify circular circACTN4 and linear ACTN4, respectively. RT-PCR revealed that circACTN4 could only be detected in cDNA but not in gDNA, whereas linear ACTN4 was amplified from both cDNA and gDNA by the convergent primers. GAPDH was considered as the reference gene (Fig. 1c). Next, the subcellular localization of circACTN4 was observed with FISH and nuclear/cytoplasmic fractionation assays. The results showed that circACTN4 was predominantly located in the nucleus of $\mathrm{BC}$ cells and the expression of circACTN4 in carcinoma tissues was higher than that in adjacent tissues (Fig. 1d and e). Moreover, qRT-PCR and RT-PCR analysis after actinomycin D treatment indicated that circACTN4 was resistant at the indicated time 


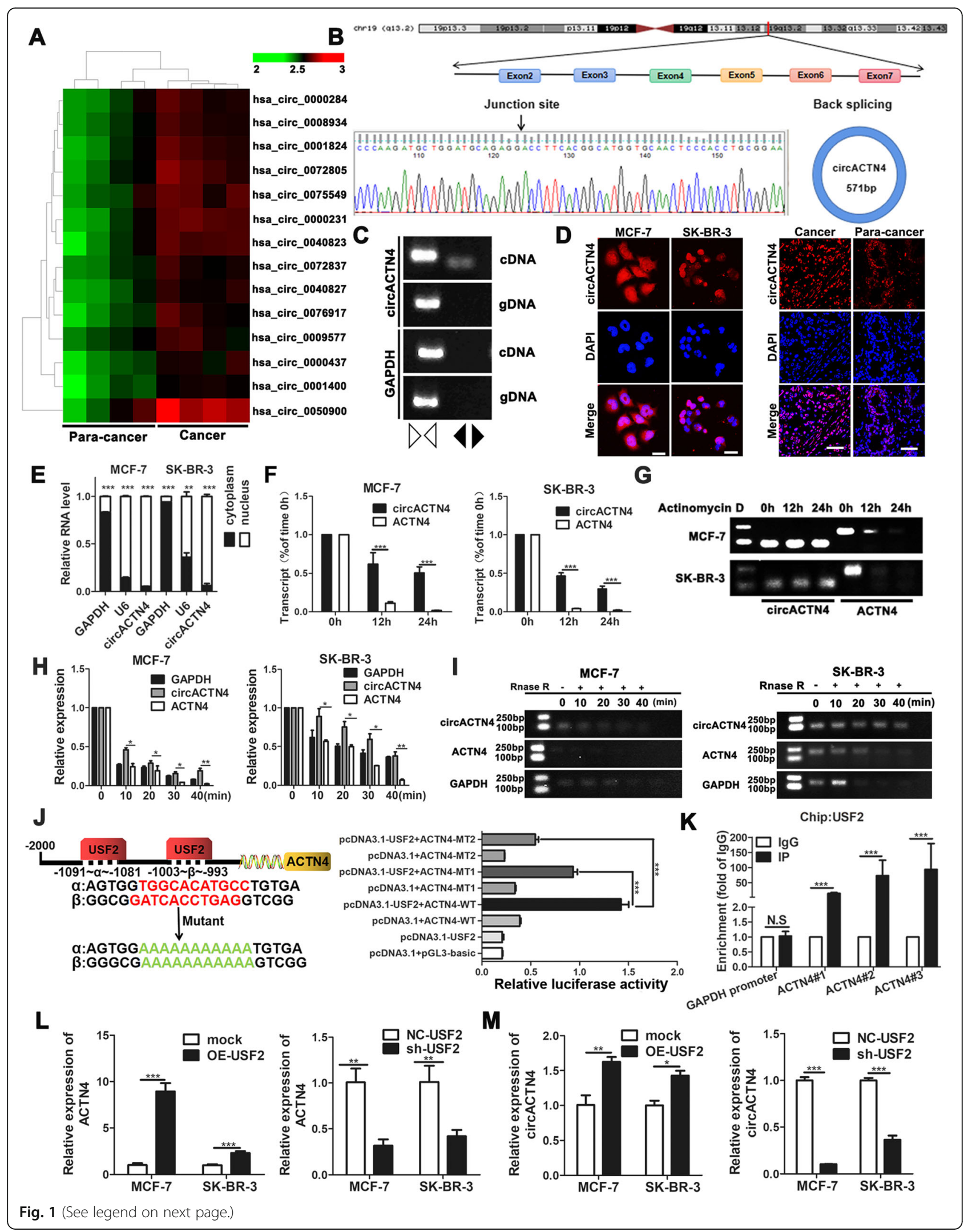




\section{(See figure on previous page.)}

Fig. 1 circACTN4 is verified and characterized in BC cells. a Heatmap showed 14 representative differentially upregulated circRNAs in BC tissues compared with adjacent normal tissues. The red and green represent the upregulated and downregulated circRNAs. $\mathbf{b}$ Schematic illustration of circACTN4 formation via the circularization from exons 2 to exon 7 in ACTN4 gene. The back-splice junction sequences were confirmed by Sanger sequencing. $\mathbf{c}$ The existence of circACTN4 from CDNA and gDNA in MCF-7 cell was detected by RT-PCR with the divergent and convergent primers. CircACTN4 could only be amplified by divergent primers from cDNA but not gDNA. $\mathbf{d}$ The localization of circACTN4 was investigated in BC cells (magnification, $\times 1000$, Scale bar, 25um) and tissues (magnification, $\times 400$, Scale bar, 50um) with FISH. e Nuclearcytoplasmic fractionation assay displayed that circACTN4 was mostly distributed in the nucleus of BC cells. U6 was considered as a nuclear control and GAPDH was used as a cytoplasmic protein control. $\mathbf{f}$ and $\mathbf{g}$ The expressions of circACTN4 and ACTN4 of BC cells were analyzed by qRT-PCR and RT-PCR after treatment with actinomycin D. $\mathbf{h}$ The levels of circACTN4 and ACTN4 were detected after RNase R digestion at different time points by qRT-PCR. i RT-PCR was used to detect the expression of circACTN4 and ACTN4 after with RNase R treatment. $\mathbf{j}$ Schematic model of wild type (WT) and mutant (Mut) sequences of two putative binding sites of USF2 on ACTN4 promoter. The relative luciferase activities were detected in MCF-7 cells co-transfected with luciferase reporter plasmids containing wild type or mutant ACTN4 promoter sequence and overexpression plasmids of USF2. $\mathbf{k}$ ChIP-qPCR analysis showed that USF2 was enriched the promoter region of ACTN4, and GAPDH promoter was used as negatively control. I and $\mathbf{m}$ The expression levels of circACTN4 and ACTN4 were determined in BC cells after USF2 up-regulation or down-regulation by qRT-PCR. GAPDH was used as the normalizing gene in the above experiments. The data are presented as the mean \pm SD, ${ }^{*} P<0.05$, ${ }^{* *} P<0.01,{ }^{* * *} P<0.001$

points, whereas linear ACTN4 mRNA transcripts were rapidly degraded (Fig.1f and g). Similarly, We further showed that circACTN4 transcripts was more stable than linear ACTN4 and GAPDH transcripts in response to RNase R digestion with qRT-PCR and RT-PCR (Fig.1h and i). Subsequently, we predicted that upstream transcription factor 2 (USF2) might bind to the promoter of ACTN4 through bioinformatics (http://jaspar.genereg. net). Thus, we inferred that USF2 could regulate the expression of ACTN4 at transcriptional level, and then we constructed luciferase reporter gene vectors carrying the full-length wt (wild type) or mut (mutant) ACTN4 promoter, the results illustrated that USF2 enhanced the luciferase activity of wild type luciferase reporter but not the mutant one (Fig. 1j). In addition, Chip-qPCR assay further proved that USF2 could bind to the ACTN4 gene promoter and enhance its transcriptional activity (Fig. 1k). Hence, the results suggested that USF2 might promote the transcriptional activity of ACTN4. Besides, to further investigate the effects of USF2 on the expression of circACTN4 in BC, we designed and constructed USF2 overexpression and knockdown plasmids, and the transfection efficiency was verified by qRT-PCR (Additional file 2: Fig. S1a). We detected that the expression of ACTN4 in BC cells transfected with the corresponding plasmids by qRTPCR. The results showed that up-regulated USF2 markedly increased expressions of linear ACTN4 and circACTN4, whereas down-regulated USF2 significantly reduced levels of linear ACTN4 and circACTN4 (Fig. 11 and $\mathrm{m}$ ). These results suggest that ACTN4 is the target gene of transcription factor USF2 .

\section{circACTN4 is highly expressed in BC tissues and cells and correlated with clinicopathological features}

To probe the expression of circACTN4 and its clinical significance, qRT-PCR was used to detect the expression of circACTN4 in 80 pairs of BC tissues and adjacent non-tumor tissues. The data showed that the expression of circACTN4 in BC tissues was significantly higher than the paired adjacent tissues (Fig. 2a), which was consistent with the results of microarray analysis. And the expression of linear ACTN4 in 20 pairs of BC and paracancer tissues were detected by qRT-PCR, data showed that linear ACTN4 was highly expressed in $\mathrm{BC}$ tissues (Additional file 2: Fig. S1g). Pearson correlation analysis displayed that the level of circACTN4 was positively related with the expression of linear ACTN4 in BC tissues (Additional file 2: Fig. S1h). Results suggest that the cooverexpression of circACTN4 and ACTN4 cooperatively promotes the progression of breast cancer. ROC (Receiver operating characteristic) curve was used to assess the diagnostic efficacy of circACTN4 in BC screening. The AUC (area under the curve) of circACTN4 was 0.719 , the diagnostic specificity and sensitivity were $70 \%$ and 70 respectively at cut-off value of 12.77 according to the ROC curve analysis (Fig. 2b). Moreover, the expression of circACTN4 was significantly higher in $\mathrm{BC}$ cells (MCF-7, SK-BR-3, ZR-75-1, T-47D and BT-474) than normal breast epithelial cell MCF-10A (Fig. 2c). Next, we assessed the association of the expression of circACTN4 with clinicalpathological features. The data revealed that the expression of circACTN4 was positively related with age $(P=0.036), \mathrm{T}(P=0.001), \mathrm{N}(P=0.001)$ and TNM stages $(P<0.001)$ but not with grade (Table 1). Subsequently, we utilized human tissue microarrays (TMAs) containing $240 \mathrm{BC}$ tissues to determine the expression of circACTN4 by ISH (Fig. 2d and e). The patients with high expression of circACTN4 had a shorter overall survival than that with low expression of circACTN4 by Kaplan-Meier survival analysis (Fig. 2f). The correlation between clinical pathological characteristics and circACTN4 expression of the $240 \mathrm{BC}$ patients was listed in Table 2. Further analysis indicated that circACTN4 expression was positive correlated with $\mathrm{T}$ stage, $\mathrm{N}$ stage and TNM stage (Fig. 2g-i). The prognostic value of circACTN4 was evaluated by Cox proportional 


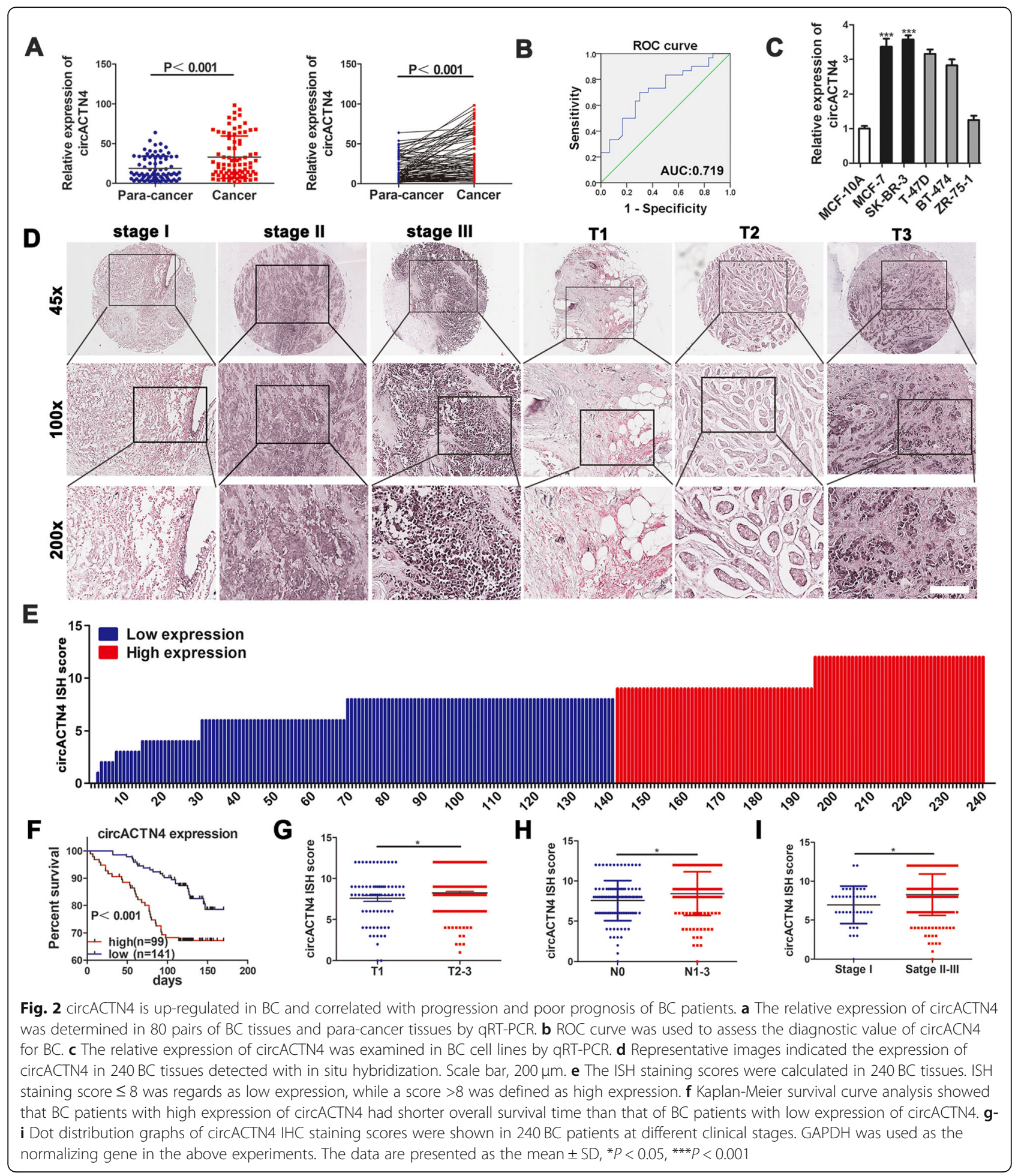

hazard model. The results showed that overexpression of circACTN4 was an independent predictor of poor prognosis in $\mathrm{BC}$ patients $(\mathrm{HR}=4.566, P<0.001)$ (Table $3)$. In brief, circACTN4 could play an oncogenic role and high expression of circACTN4 could predict poor prognosis in $\mathrm{BC}$ patients.
circACTN4 enhances proliferation and regulates apoptosis of $B C$ cells

To explore the biological function of circACTN4 in BC cells, circACTN4 overexpression plasmid and small interference RNAs (siRNAs) targeting circACTN4 were transfected into $\mathrm{BC}$ cells. The results indicated that the 
Table 1 Correlation between circACTN4 expression and clinicopathological features in $80 \mathrm{BC}$ patients (cohort 1)

\begin{tabular}{|c|c|c|c|c|c|c|}
\hline \multirow[t]{2}{*}{ Characteristic } & & \multirow{2}{*}{$\begin{array}{l}\text { All } \\
\text { cases }\end{array}$} & \multicolumn{2}{|c|}{ CircACTN4 } & \multirow{2}{*}{$\begin{array}{l}\text { Chi- } \\
\text { square }\end{array}$} & \multirow[t]{2}{*}{$P$ value } \\
\hline & & & low & high & & \\
\hline All cases & & 80 & 18 & 62 & & \\
\hline Age & $\begin{array}{l}<53 \\
\geq 53\end{array}$ & $\begin{array}{l}45 \\
35\end{array}$ & $\begin{array}{l}14 \\
4\end{array}$ & $\begin{array}{l}31 \\
31\end{array}$ & 4.374 & $0.036^{*}$ \\
\hline Grade & $\begin{array}{l}\| \\
I I I\end{array}$ & $\begin{array}{l}17 \\
63\end{array}$ & $\begin{array}{l}5 \\
13\end{array}$ & $\begin{array}{l}12 \\
50\end{array}$ & 0.591 & 0.442 \\
\hline T stage & $\begin{array}{l}\text { T1 } \\
\text { T2-3 }\end{array}$ & $\begin{array}{l}35 \\
45\end{array}$ & $\begin{array}{l}14 \\
4\end{array}$ & $\begin{array}{l}21 \\
41\end{array}$ & 10.928 & $0.001^{* *}$ \\
\hline $\mathrm{N}$ stage & $\begin{array}{l}\text { No } \\
\text { N1-3 }\end{array}$ & $\begin{array}{l}40 \\
40\end{array}$ & $\begin{array}{l}15 \\
3\end{array}$ & $\begin{array}{l}25 \\
37\end{array}$ & 10.323 & $0.001^{* *}$ \\
\hline TNM stage & $\begin{array}{l}\text { I } \\
\|/\| I \|\end{array}$ & $\begin{array}{l}23 \\
57\end{array}$ & $\begin{array}{l}14 \\
4\end{array}$ & $\begin{array}{l}9 \\
53\end{array}$ & 27.254 & $<0.001^{* * *}$ \\
\hline
\end{tabular}

${ }^{*} P<0.05,{ }^{* *} P<0.01,{ }^{* * *} P<0.001$

expression of circACTN4 was remarkably up-regulated or down-regulated in $\mathrm{BC}$ cells transfected with the corresponding vectors or siRNAs by qRT-PCR (Fig. 3a). However, both overexpression and knockdown of circACTN4 did not impact on the expression level of linear transcript ACTN4 with qRT-PCR (Fig. 3b). The colony formation, EdU and CCK-8 assays showed that overexpression of circACTN4 markedly enhanced the proliferation ability of $\mathrm{BC}$ cells, whereas knockdown of circACTN4 significantly inhibited cell viability (Fig. 3cg). Moreover, hoechst 33342 staining showed that $\mathrm{BC}$ cells appeared obvious morphological feature of apoptosis including stronger fluorescenc, enuclear fragment, chromatin aggregation and apoptosis body after transfected with si-circACTN4 (Fig. 3h). In addition, the apoptosis rate was detected by flow cytometry using AnnexinV/PI-staining, and the results indicated that circACTN4 knockdown could induce apoptosis of BC (Fig. 3i). Finally, we detected the apoptosis-related proteins by western blot, results showed that down-regulation of

Table 2 Correlation between circACTN4 expression and clinicopathological features in 240 BC patients (cohort 2)

\begin{tabular}{|c|c|c|c|c|c|c|}
\hline \multirow[t]{2}{*}{ Characteristic } & & \multirow{2}{*}{$\begin{array}{l}\text { All } \\
\text { cases }\end{array}$} & \multicolumn{2}{|c|}{ CircACTN4 } & \multirow{2}{*}{$\begin{array}{l}\text { Chi- } \\
\text { square }\end{array}$} & \multirow{2}{*}{$\begin{array}{l}P \\
\text { value }\end{array}$} \\
\hline & & & low & high & & \\
\hline All cases & & 240 & 141 & 99 & & \\
\hline Age & $\begin{array}{l}<53 \\
\geq 53\end{array}$ & $\begin{array}{l}118 \\
122\end{array}$ & $\begin{array}{l}68 \\
73\end{array}$ & $\begin{array}{l}50 \\
49\end{array}$ & 0.121 & 0.728 \\
\hline Grade & $\begin{array}{l}\| \\
\text { III }\end{array}$ & $\begin{array}{l}190 \\
50\end{array}$ & $\begin{array}{l}114 \\
27\end{array}$ & $\begin{array}{l}76 \\
23\end{array}$ & 0.588 & 0.443 \\
\hline T stage & $\begin{array}{l}\text { T1 } \\
\text { T2-3 }\end{array}$ & $\begin{array}{l}73 \\
167\end{array}$ & $\begin{array}{l}50 \\
91\end{array}$ & $\begin{array}{l}23 \\
76\end{array}$ & 4.109 & $0.043^{*}$ \\
\hline N stage & $\begin{array}{l}\text { No } \\
\text { N1-3 }\end{array}$ & $\begin{array}{l}108 \\
132\end{array}$ & $\begin{array}{l}76 \\
65\end{array}$ & $\begin{array}{l}32 \\
67\end{array}$ & 10.941 & $0.001^{* *}$ \\
\hline TNM stage & $\left.\right|_{\|/\| I \mid}$ & $\begin{array}{l}40 \\
200\end{array}$ & $\begin{array}{l}30 \\
111\end{array}$ & $\begin{array}{l}10 \\
89\end{array}$ & 5.230 & $0.022^{*}$ \\
\hline
\end{tabular}

${ }^{*} P<0.05,{ }^{* *} P<0.01$
circACTN4 led to higher levels of activated (cleaved) caspase-3 and proapoptotic protein Bax and a lower expression of Bcl-2 compared with control group (Fig. 3j).

\section{circACTN4 increases migration and invasion and modulates cell cycle progression of $\mathrm{BC}$ cells}

To further assess the role of circACTN4 in BC progression, migration, invasion and cell cycle of $\mathrm{BC}$ cells were investigate after overexpression or knockdown of circACTN4. The wound healing and transwell assays indicated that the invasive and migratory abilities of $\mathrm{BC}$ cells were remarkably increased by up-regulation of circACTN4 but significantly suppressed by downregulation of circACTN4 (Fig. 4a-f). Furthermore, the expressions of nm23-H1, MMP9 and MMP2 were detected by western blot, the results showed that MMP9 and MMP2 protein levels were obviously enhanced or reduced and nm23-H1 expression was markedly decreased or increased in $\mathrm{BC}$ cells after overexpression or knockdown of circACTN4 (Fig. 4g and h). Moreover, cell cycle analysis was determined by flow cytometry, the results revealed that knockdown of circACTN4 led to lower percentage of $\mathrm{BC}$ cells in $\mathrm{S}$ phase and higher proportion of $\mathrm{BC}$ cells in $\mathrm{G} 1$ phase compared with control group, which suggest that circACTN4 silencing caused G1 arrest of BC cells (Fig. 4i). Additionally, the expression of cell cycle- related proteins was measured with western blot, we found that CDK4, CCNE1 and CCND1 protein levels were significantly downregulated after knockdown of circACTN4 in BC cells, which might prevent cell cycle progression of BC cells (Fig. 4j).

\section{CircACTN4 directly interacts with FUBP1 and activates transcription of MYC}

To explore the molecular mechanism underlying circACTN4, we first performed pull down, followed by silver staining and mass spectrometry analysis to detect the circACTN4-binding proteins, we found that FUBP1 was significantly enriched on circACTN4 (Fig. 5a and b). Subsequently, RIP assay further confirmed that FUBP1 could specifically combine with endogenous circACTN4 in BC cells by PCR and qRT-PCR (Fig. 5c). FISH-IF assay displayed that circACTN4 and FUBP1 were colocalized in the nucleus (Fig. 5d). But upregulation and knockdown of circACTN4 did not alter FUBP1 expression levels (Fig. 5e), suggesting that circACTN4 was not involved in the post-translational regulation of FUBP1. Previous studies have shown that FUBP1 could bind to the FUSE region of the MYC promoter to promote the expression of MYC, while FUBP1-FUSE complex might recruit FIR to inhibit MYC transcription. Next, ChIPPCR assay confirmed that FUBP1 binds to FUSE of the MYC promoter in $\mathrm{BC}$ cells (Fig. $5 \mathrm{f}$ ). To explore the effects of FUBP1 and FIR in BC, we constructed FUBP1 
Table 3 Univariate and multivariate Cox regression analysis of circACTN4 and survival in 240 BC patients

\begin{tabular}{|c|c|c|c|c|c|c|}
\hline \multirow[t]{2}{*}{ Clinical variables } & \multicolumn{2}{|c|}{ Univariate analysis } & \multirow[t]{2}{*}{$P$} & \multicolumn{2}{|c|}{ Multivariate analysis } & \multirow[t]{2}{*}{$P$} \\
\hline & HR & $95 \% \mathrm{Cl}$ & & HR95 & & \\
\hline Age ( $\geq 53$ vs. < 53) & 1.757 & $1.079-2.860$ & $0.023^{*}$ & 1.894 & $1.161-3.089$ & $0.011^{*}$ \\
\hline Grade (II vs. III) & 1.982 & $1.192-3.294$ & $0.008^{* *}$ & 1.989 & $1.194-3.316$ & $0.008^{* *}$ \\
\hline T stage (T1 vs. T2/3) & 1.383 & $0.800-2.392$ & 0.246 & & & \\
\hline stage (N0-1 vs. N2-3) & 2.234 & $1.327-3.758$ & $0.002^{* *}$ & 1.499 & $0.868-2.591$ & 0.147 \\
\hline TNM stage (I vs. II/III) & 2.937 & $1.182-7.299$ & $0.020^{*}$ & 1.972 & $0.766-5.077$ & 0.159 \\
\hline CircACTN4 (low vs. high) & 5.040 & $2.913-8.720$ & $<0.001^{* * *}$ & 4.566 & $2.612-7.982$ & $<0.001^{* * *}$ \\
\hline
\end{tabular}

Abbreviations: $H R$ hazard ratio, $\mathrm{Cl}$ confidence interval

${ }^{*} P<0.05,{ }^{* *} P<0.01,{ }^{* * *} P<0.001$

and FIR overexpression and knockdown plasmids. The transfection efficiencies were determined by qRT-PCR and western blot. (Additional file 2: Fig. S1b-e). The results of qRT-PCR and western blot demonstrated that upregulation or downregulation of FUBP1 evidently increased or decreased expression levels of MYC in BC cells, respectively (Fig. $5 \mathrm{~g}$ and h). Moreover, we found that the expression of FUBP1 in BC tissues was noticeably higher than that in adjacent normal tissues by qRTPCR (Fig. 5i). Pearson correlation analysis showed that the level of circACTN4 was positively related with the expression of FUBP1 in BC tissues (Fig. 5j). Furthermore, MYC was highly expressed in $\mathrm{BC}$ tissues and positively correlated with the expression of FUBP1 (Fig. 5k and $\mathrm{l}$ ). We also demonstrated that there was a positive correlation between the expression of circACTN4 and MYC by qRT-PCR and Pearson correlation analysis (Fig. $5 \mathrm{~m}$ and $\mathrm{n})$. In addition, some studies have shown that ACTN4 can also promote the progression of breast cancer. To confirm the biological function of ACTN4 in $\mathrm{BC}, \mathrm{ACTN} 4$ overexpression and interference plasmids were constructed, the transfection efficiencies were determined by qRT-PCR (Additional file 2: Fig. S1i). And then, to probe that the effect of circACTN4 is independent of ACTN4, co-transfection experiments of circACTN4 or si-circ\#2 and sh-ACTN4 or OEACNT4 were conducted, the results showed that ACTN4 knockdown did not affect the promoting role of overexpressed circACTN4 on MYC transcription, and upregulation of ACTN4 did not reverse the inhibitory effect of circACTN4 knockdown on MYC expression by $\mathrm{qRT}-\mathrm{PCR}$ and western blot (Additional file 2: Fig. $\mathrm{S} 1 \mathrm{j}$ and $\mathrm{k}$ ). Additionally, the results of western blot showed that circACTN4 could promote the expressions of MYC and its downstream proteins CDK4 and CCND2 (Fig. 5o). However, whether circACTN4 was up-regulated or down regulated in $\mathrm{BC}$ cells, there was no effect on the protein levels of FUBP1 and FIR (Additional file 2: Fig. S1f). Together, these data suggest that circACTN4 could bind to FUBP1 and promote the expression of MYC.

\section{circACTN4 and FIR competitively combine with FUBP1}

Therefore, we presume that circACTN4 could competitively combine with FUBP1 and prevent the binding of FIR to FUBP1 to promote the transcription and expression of MYC. In order to further verify proposed hypothesis, we first detected the expression of FIR in BC issues and matched normal tissues by qRT-PCR, and our data revealed that the expression of FIR was significantly downregulated in $\mathrm{BC}$ tissues compared with normal adjacent tissues (Fig. 6a). Pearson correlation analysis indicated that the expression of FIR was negatively associated with the levels of circACTN4, FUBP1 and MYC in BC tissues (Fig. 6b-d). Subseqently, we explored whether circACTN4 could influence the binding between FIR and FUBP1. Co-immunoprecipitation assays were performed in MCF-7 cells with anti-FUBP1 antibody. The results showed that no obvious FIR protein band was found in the FUBP1-co-immunocoprecipitates of BC cells overexpressing circACTN4, whereas FIR was clearly detected in the co-immunocoprecipitates by western blot after circACTN4 was knocked down, which suggest upregulated circACTN4 remarkably weakened the binding of FIR to FUBP1 while downregulated circACTN4 significantly strengthened the interaction between FUBP1 and FIR (Fig. 6e). A series of ChIP assay results showed that the binding level of FIR and FUSE after transfection with high concentration of si-circ\#2 was higher than that of FIR and FUSE after transfection with low concentration of si-circ\#2 (Fig. 6f), but had no effect on the binding level of FUBP1 and FUSE after transfection with high or low concentration of si-circ\#2 (Additional file 2: Fig. S1l). Furthermore, we found that silencing FIR markedly enhanced the expression of MYC, whereas overexpressing FIR decreased the level of MYC by qRT-PCR and western blot in BC cells (Fig. $6 \mathrm{~g}$ and h). Finally, after co-transfection of OE-FIR or sh-FIR with circACTN4 or si-circ\#2, we found that overexpression FIR could reverse the enhancing effect of circACTN4 on MYC expression, while FIR knockdown could counteract the inhibitory impact of downregulation of circACTN4 on MYC level by qRT-PCR (Fig. 6i). 
A

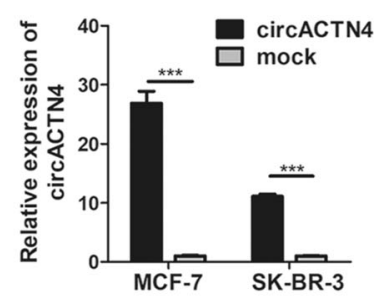

C

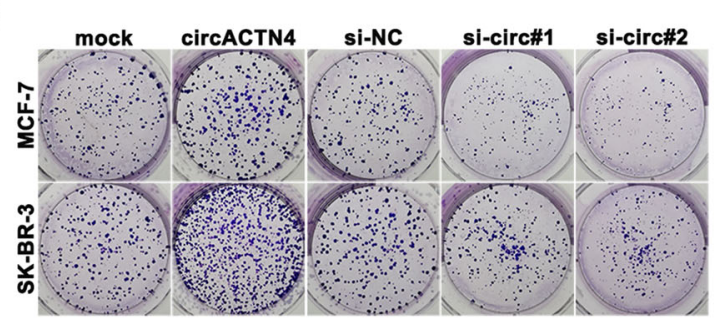

E

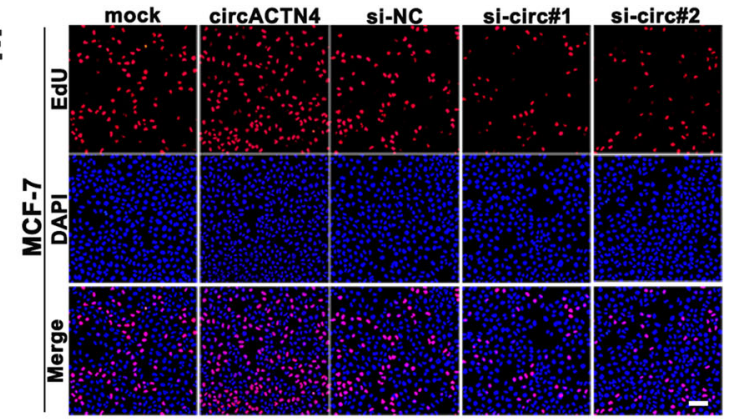

F
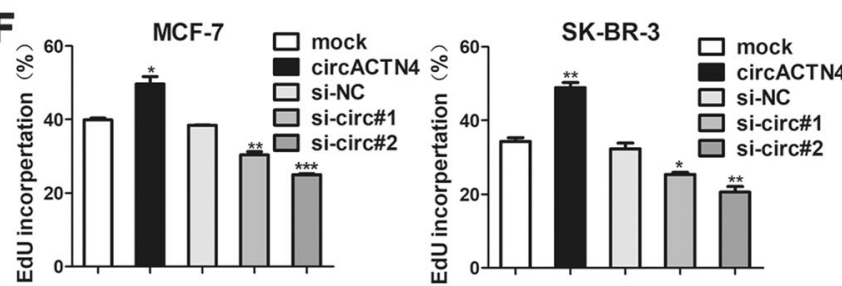

D
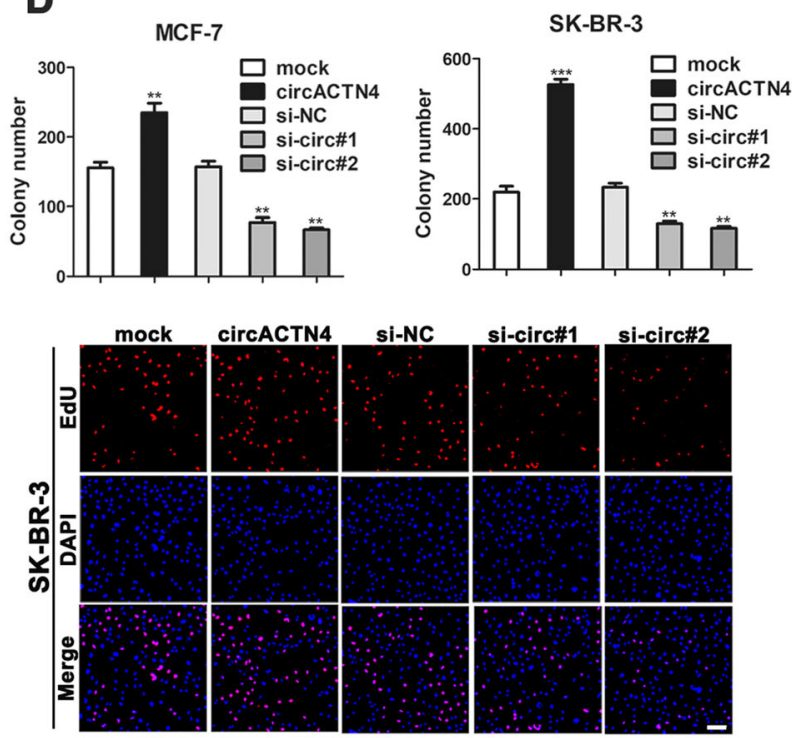

circACTN4 si-NC

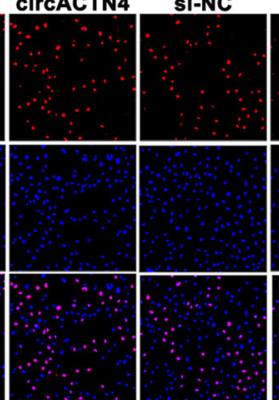

G ${ }_{4} \quad \mathrm{MCF}-7$
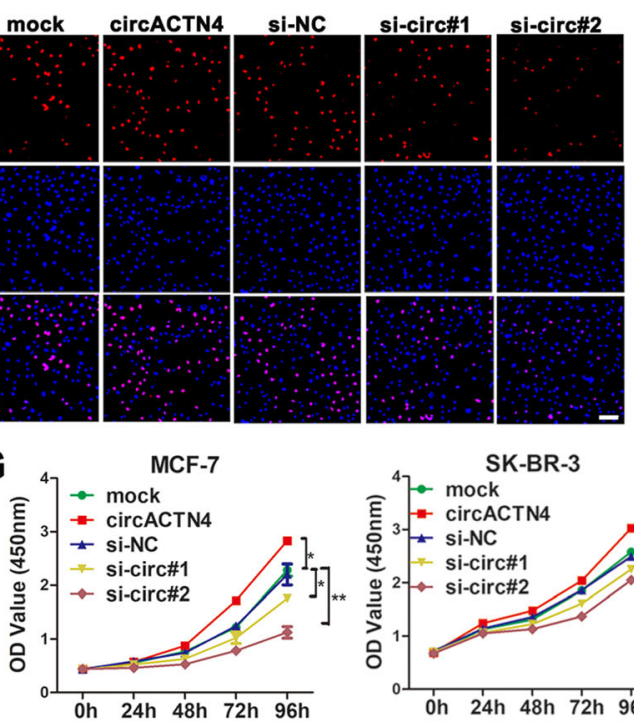

SK-BR-3
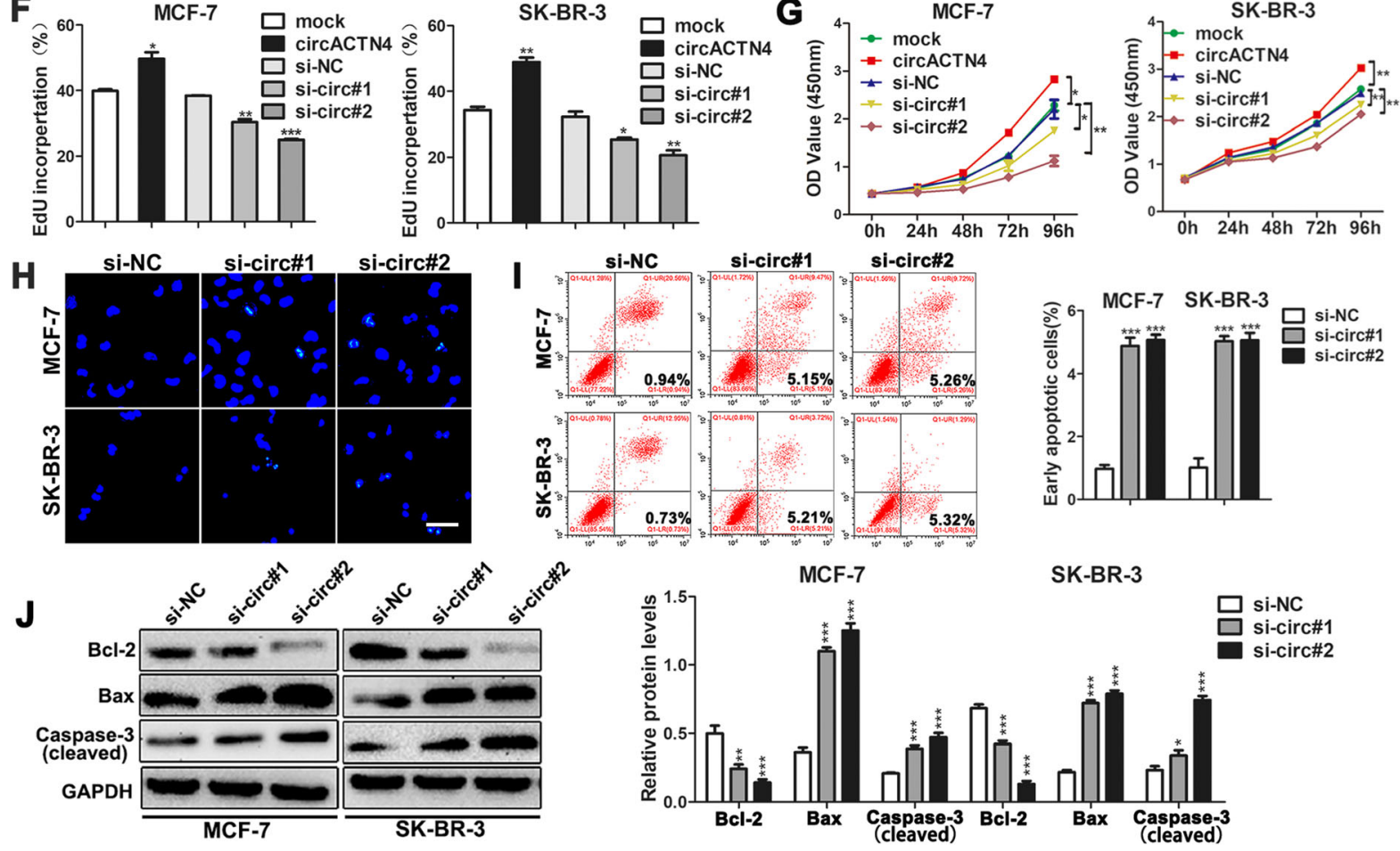

Fig. 3 (See legend on next page.) 
(See figure on previous page.)

Fig. 3 circACTN4 increases proliferation and suppresses apoptosis of BC cells. $\mathbf{a}$ and $\mathbf{b}$ The relative expressions of circACTN4 and ACTN4 were detected in BC cells after transfection with overexpressed cirCACTN4 plasmid or si-circACTN4 by qRT-PCR. $\mathbf{c}$ and $\mathbf{d}$ Colony formation assay was used to assess cell survival in BC cells transfected with indicated plasmids and siRNA. $\mathbf{e}$ and $\mathbf{f}$ EdU assay was conducted to detect cell proliferation ability of BC cells (magnification, $\times 100$, Scale bar, 100um). $\mathbf{g}$ CCK-8 assay was performed to evaluate cell viability. $\mathbf{h}$ Morphological changes of apoptotic cells were observed by Hoechst 33342 staining (magnification, $\times 400$, Scale bar, 50um) after knockdown of circACTN4. i The apoptosis rate was analyzed by flow cytometry after downregulation of circACTN4. $\mathbf{j}$ The expressions of apoptosis-related proteins were determined in BC cells transfected with indicated siRNA by Western blot. GAPDH was used as the normalizing gene in the above experiments. The data are presented as the mean $\pm \mathrm{SD},{ }^{*} P<0.05,{ }^{* *} P<0.01,{ }^{* *} P<0.001$

In summary, these results demonstrated that circACTN4 could inhibit the binding FIR and FUBP1 to relieve the repressive effect of FIR, thereby promoting MYC transcription.

\section{FIR reverses the tumor promoting-effects of circACTN4 in $B C$ cells}

To further investigate whether circACTN4 plays its biological role via circACTN4/FUBP1/MYC axis, a series of rescue experiments were executed in $\mathrm{BC}$ cells after cotransfection of OE-FIR or sh-FIR with circACTN4 or sicirc\#2. The results show that FIR overexpression could markedly reverse the promoting roles of circACTN4 upregulation in proliferation, migration and invasion of $\mathrm{BC}$ cells, while FIR knockdown attenuated the inhibitory effects mediated by circACTN4 downregulation in BC cells by wound healing, EdU and transwell assays (Fig. 7a-d). Moreover, IF assay also showed that FIR overexpression and knockdown could evidently reverse the effects of upregulation and downregulation of circACTN4 on MYC expression (Fig. 7e and f). Additionally, compared with para-cancer tissues, we found that MYC was highly expressed in BC tissues by IF (Fig. 7g). Furthermore, western blot analysis showed that upregulation and downregulation of FIR could counteract the enhancing and suppressing effects on the expression of MYC and its downstream protein CDK4 and CCND2 by overexpressing and silencing circACTN4 (Fig. 7h). Collectively, the above results further proved that circACTN4 might competitively bind with FUBP1 to block the transcriptional inhibitory effect of FIR on MYC, which could promote to tumorigenesis and progression of $\mathrm{BC}$.

\section{CircACTN4 facilitates tumorigenesis and metastasis of xenograft tumors in vivo}

To evaluate the oncogenic effect of circACTN4 in vivo, the tumor models of human $\mathrm{BC}$ cells were established in nude mice by subcutaneous injection and caudal vein injection. Female nude mice were inoculated with the MCF-7 cells infected with circACTN4 overexpressing lentiviruses or circACTN4 shRNA lentiviruses and their controls. The results indicated that the volume and weight of xenograft tumor in overexpression circACTN4 group were markedly larger than those in the control group, whereas circACTN4 knockdown significantly suppressed the tumor growth (Fig. 8a-c). Furthermore, compared with control group, the upregulation of circACTN4 obviously increased the number of metastatic pulmonary nodules, while circACTN4 silencing significantly repressed spontaneous pulmonary metastasis with less invasive tumor cells (Fig. 8d). Moreover, the overexpression of circACTN4 could remarkably facilitate tumor angiogenesis, whereas circACTN4 knockdown markedly reduced tumor microvessel density (Fig. 8e). Next, the results of western blotting revealed that the protein level of MYC in the xenograft tumor tissue from overexpressing or silencing circACTN4 group was significantly increased or decreased, respectively compared with the control group (Fig. 8f). Subsequently, to further observe the impact of circACTN4 on tumor metastasis in vivo, stable circACTN4-overexpressing and circACTN4-silencing MCF-7 cells were intravenously injected into the tail vein of BALB/c nude mice. The micrometastases were monitored using bioluminescent imaging (BLI). The results displayed that stronger and more bioluminescent signals were detected in the circACTN4 overexpressing group, while mice in circACTN4 silencing group displayed smaller the number of bioluminescent metastases compared with the control group (Fig. 8g). In addition, the mice in the circACTN4 overexpressing group had lower overall survival rate and more extensive liver metastasis compared with the control group (Fig. $8 \mathrm{~h}$ and i). Finally, we determined the effects of circACTN4 on its target protein MYC, downstream cell cycle related proteins CDK4 and CCND2 as well as cell proliferation related protein Ki67 by IHC. The results showed that upregulation of circACTN4 could increase the expressions of MYC, CDK4, CCND2 and Ki67 in tumor tissues, whereas circACTN4 silencing reduced the expression levels of these proteins (Fig. 8j). Together, these above results further demonstrated the oncogenic role of circACTN4 in BC, suggesting that circACTN4 could promote development and metastasis of breast cancer through activating protooncogene MYC.

\section{Discussion}

Breast cancer is a heterogeneous and complex disease with the high incidence and the mortality in female 


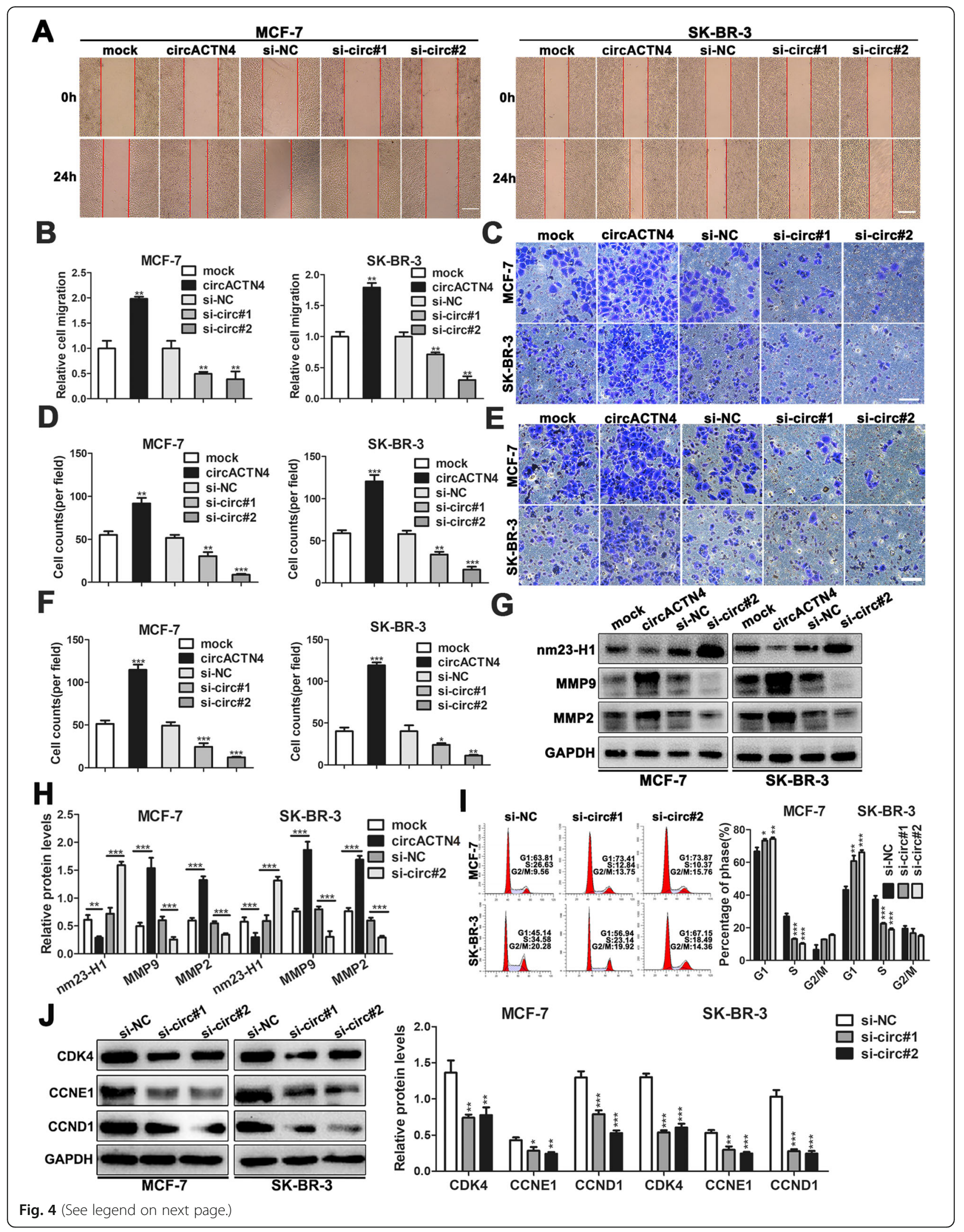


(See figure on previous page.)

Fig. 4 cirCACTN4 promotes migration, invasion and cell cycle progression of BC cells. $\mathbf{a}$ and $\mathbf{b}$ The migration ability of BC cells were detected by wound healing assays (magnification, $\times 50$, Scale bar, 200um) after overexpression or knockdown of circACTN4. c-f Transwell assay was used to measure BC cells invasion and migration abilities after upregulation and downregulation of circACTN4, respectively (magnification, $\times 100$, Scale bar, $100 \mathbf{u m}) . \mathbf{g}$ and $\mathbf{h}$ The expressions of $\mathbf{n m} 23-\mathrm{H1}$, MMP9 and MMP2 were detected in BC cells transfected with circACTN4 overexpression plasmids and si-circ\#2 by western blot. $\mathbf{i}$ Cell cycle analysis was executed by flow cytometry after circACTN4 silencing. $\mathbf{j}$ The expressions of cell cycle-related proteins were detected in BC cells transfected with indicated siRNA by western blot. GAPDH was used as the normalizing gene in the above experiments. The data are presented as the mean $\pm S D,{ }^{*} P<0.05,{ }^{* *} P<0.01,{ }^{* *} P<0.001$

worldwide. Though some breast cancer patients reveal good prognosis, there are still some patients with tumor progression, which has become a threat to public health [22]. Emerging evidence suggests that circRNAs play pivotal roles in the tumorigenesis and development of diverse human cancers [2]. However, the molecular mechanism of circRNAs in the progression of breast cancer is largely unknown. In this study, we found that circACTN4 (hsa_circ_0050900), a novel nuclear circRNA, was significantly overexpressed in breast cancer and was closely related to advanced clinical stage, metastasis and poor prognosis of $\mathrm{BC}$ patients. Functionally, we showed that up-regulation of circACTN4 promoted the growth and aggressiveness of breast cancer cells in vitro and in vivo, while down-regulation of circACTN4 had the contrary effects. Mechanistically, we found that USF2 could accelerate the biogenesis of circACTN4. More importantly, we discovered that circACTN4 could competitively bind to FUBP1 and block the connection between FUBP1 and FIR, thus stimulating MYC transcription. Our study demonstrate that circACTN4 is a novel oncogenic circRNA in breast cancer through activation of MYC and plays an important role in the progression of breast cancer.

Most circRNAs were reported to locate in the cytoplasm and work as miRNA sponge, which regulate tumor progression via competing endogenous RNA (ceRNA) mechanism [23]. However, some circRNAs are predominantly expressed in the nucleus to combine with RNA-binding proteins and regulate their activity. For example, circCUX1 binds with EWS RNA-binding protein 1 (EWSR1) to promote its interplay with MAZ (MYCassociated zinc finger protein), glycolysis and neuroblastoma progression [24]. Furthermore, circ-CTNNB1 binding DEAD-box polypeptide 3 (DDX3) promotes its physical interaction with transcription factor Yin Yang 1 (YY1) [25]. Additionally, circ-DONSON might recruit the NURF complex to transcription factor SOX4 promoter and initiate its transcription, thus facilitating gastric cancer growth and invasion [26]. In our study, we revealed that circACTN4 was primarily located in the nucleus of BC cells, which suggested that circACTN4 might be not a miRNA sponge. The function and mechanism of circRNAs are related to their subcellular localization pattern [27]. We demonstrated that circACTN4 was involved in transcription of MYC, which occurs in nucleus and control cell proliferation [28]. Up to now, how circRNAs exert functions in the nucleus remains elusive.

FUBP1, an ATP dependent DNA helicase V, is a significant regulator of transcription, translation and RNA splicing by binding with single stranded DNA (ssDNA) and RNA. FUBP1 binds to the FUSE (far upstream element) of c-myc promoter and activates transcription of cmyc by TFIIH helicase activity. FIR could inhibit the activation effect of FUBP1. The interaction of FUBP1 with FIR and TFIIH affected the time and the level of MYC gene expression. Therefore, FUSE/FUBP1/FIR complex provides a fine-tuned regulation of MYC $[29,30]$. Emerging evidence has showed both the oncogenic and suppressive roles of FUBP1. Mutations in the FUBP1 gene and loss of function are frequently found in patients with central nervous system diseases and intestinal cancer $[17,31]$. Whereas, overexpression of FUBP1 is also often appears in various cancers including breast cancer, gastric cancer, hepatocellular carcinoma, nasopharyngeal carcinoma and leukemia [19, 32-35]. High expression of FUBP1 usually leads to upregulation of c-MYC oncogene and disorder of the fine regulation of target proteins, which is one of main molecular mechanisms of tumor pathogenesis. For example, there is a positive correlation between the expression of FUBP1 and c-MYC in glioma and high expressions of FBP1 and c-MYC are associated with poor prognosis [36]. FUBP1 stimulates c-MYC expression in esophageal squamous cell carcinoma (ESCC) and facilitates ESCC development [37]. Sun. et al. demonstrated that lncRNA SNHG1 regulated the expression of MYC by interacting with FUBP1 and preventing the binding of FUBP1 and FIR, thus blocking FIR mediated MYC transcription inhibition [20]. Consistent with the previous reports, we found that FUBP1 was highly expressed in $\mathrm{BC}$ tissues, and further demonstrated circACTN4 could directly bind to FUBP1, we also found a positive correlation between the expression of circACTN4 and MYC in BC. Besides, we found that the expression of FIR was negatively correlated with expression of MYC. Moreover, upregulated circACTN4 inhibited the combination of FUBP1/FUSE complex 


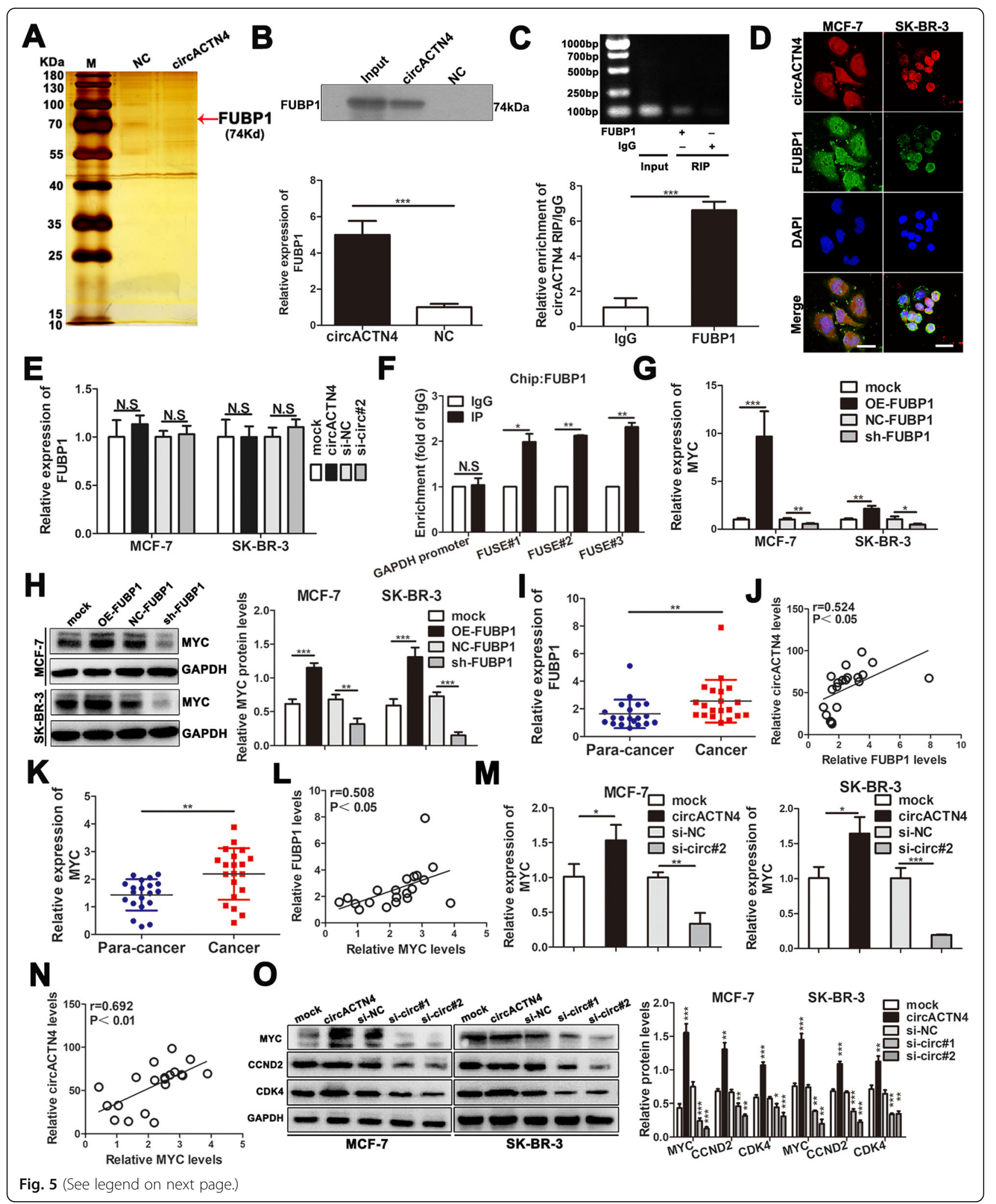


(See figure on previous page.)

Fig. 5 circACTN4 can bind to FUBP1 to upregulate MYC expression. a and b RNA pull-down experiment was performed using the specific biotinlabeled circACTN4 probe in MCF-7 cell lysates, followed by silver staining, and the protein bands were analyzed by mass spectrometry and western blot. FUBP1 was identified as a candidate protein interacting with circACTN4. c RIP assay was executed in MCF-7 cell lysates using antiFUBP1or anti-IgG, then the enrichment of circACTN4 was detected by RT-PCR and GRT-PCR. $\mathbf{d}$ FISH-IF assay displayed that circACTN4 was COlocalized with FUBP1 in the nucleus of BC cells (magnification, $\times 1000$, Scale bar, 25um). e The relative expression of FUBP1 was determined in the BC cells transfected with overexpressed circACTN4 plasmid or si-circACTN4 by qRT-PCR. $\mathbf{f}$ Chip assay showed that FUBP1 was enriched in the FUSE upstream of MYC promoter and was not enriched in the GAPDH promoter. $\mathbf{g}$ and $\mathbf{h}$ The effects of overexpression and knockdown of FUBP1 in BC cells on the expression of MYC were detected by qRT-PCR and western blot. $\mathbf{i}$ The relative expression of FUBP1 in 20 pairs of BC tissues and para-cancer tissues was determined by qRT-PCR. $\mathbf{j}$ Pearson correlation analysis showed that the expression of FUBP1 was positively correlated with the level of circACTN4. $\mathbf{k}$ The relative expression of MYC in 20 pairs of BC tissues and para-cancer tissues was detected by qRTPCR. I Pearson correlation analysis showed that the expression of FUBP1 was positively correlated with that of MYC. $\mathbf{m}$ The relative expression of MYC was evaluated after transfection with overexpressed circACTN4 plasmid and si-circACTN4 by qRT-PCR. $\mathbf{n}$ The expression of circACTN4 was positively associated with that of MYC by pearson correlation analysis. o The expressions of MYC and its downstream proteins CDK4 and CCND2 were assessed in the BC cells transfected with overexpressed circACTN4 plasmid and si-circACTN4 by western blot. GAPDH was used as the normalizing gene in the above experiments. The data are presented as the mean $\pm \mathrm{SD},{ }^{*} P<0.05$, ${ }^{* *} P<0.01,{ }^{* *} P<0.001$

and FIR, while downregulated circACTN4 exerted the opposite effect. These results confirmed that circACTN4 might directly interacted with FUBP1 to prevent the binding of FUBP1 to FIR, thus promoting the expression of the oncogene $\mathrm{MYC}$ and progression of $\mathrm{BC}$.
MYC play an important oncogenic role in nearly every aspect of biological processes including proliferation, differentiation, apoptosis and metabolism. MYC is frequently deregulated in human cancers and associated with poor prognosis. It is estimated that its expression is

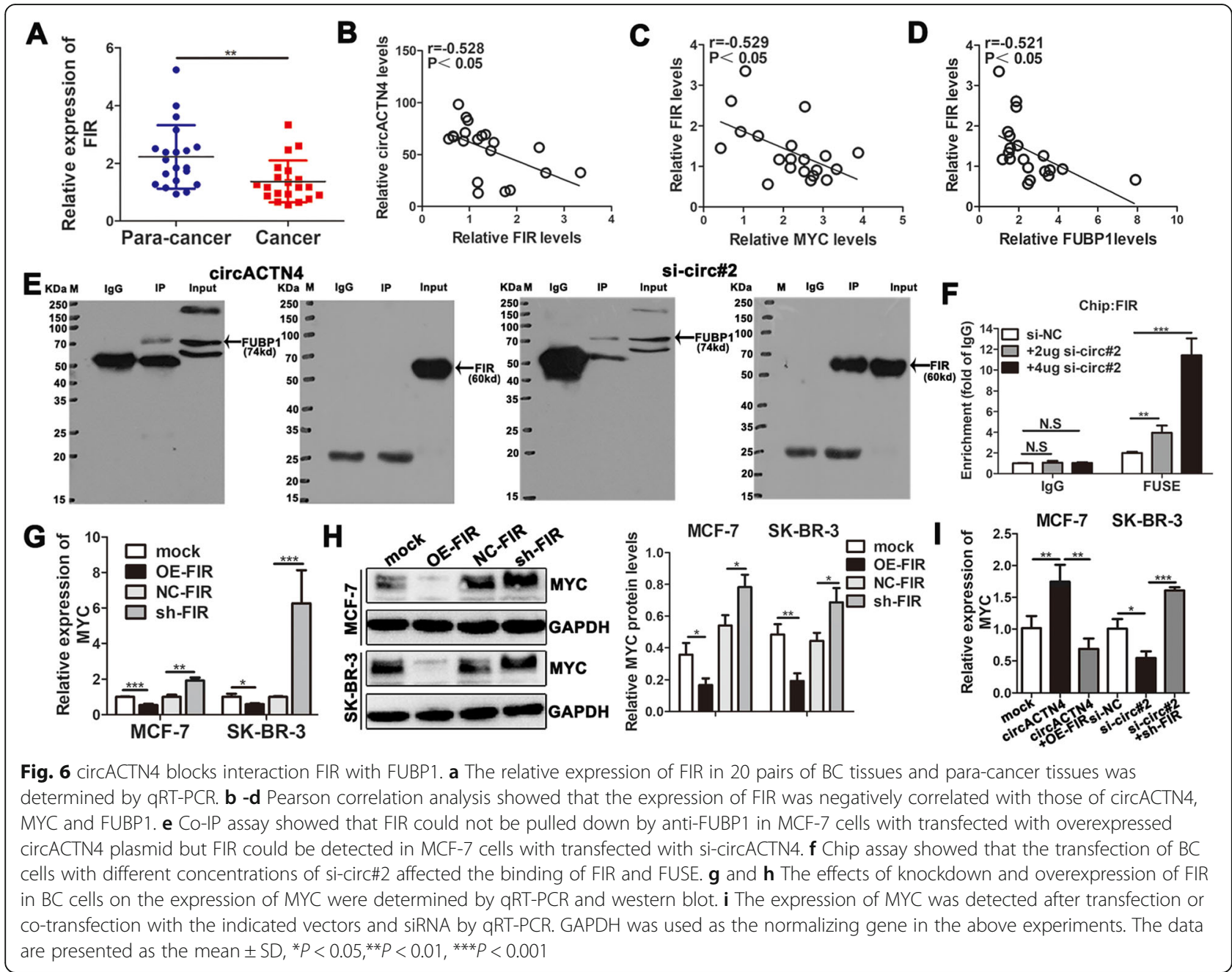



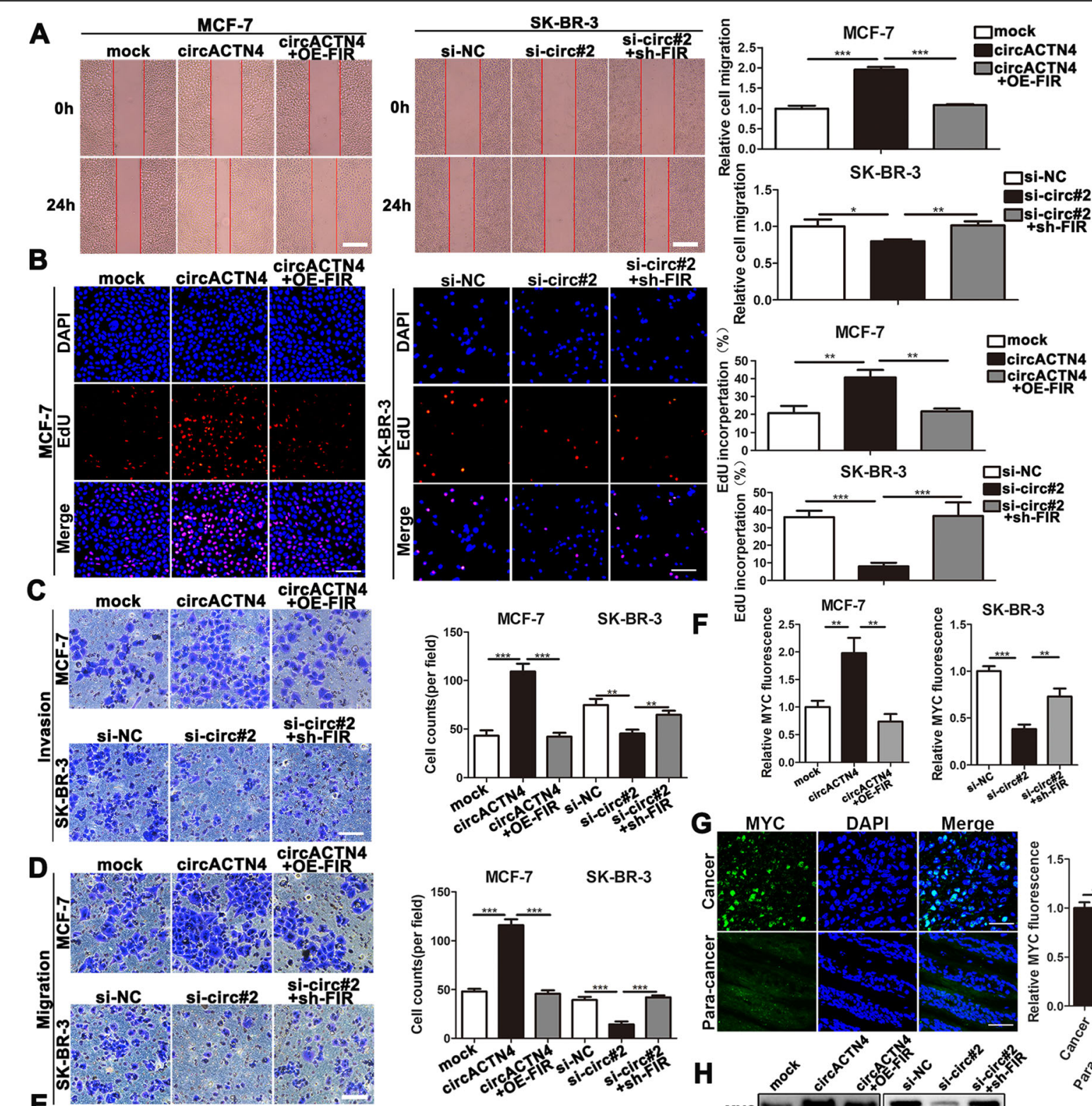

$E^{\mid c o ̛ s}$

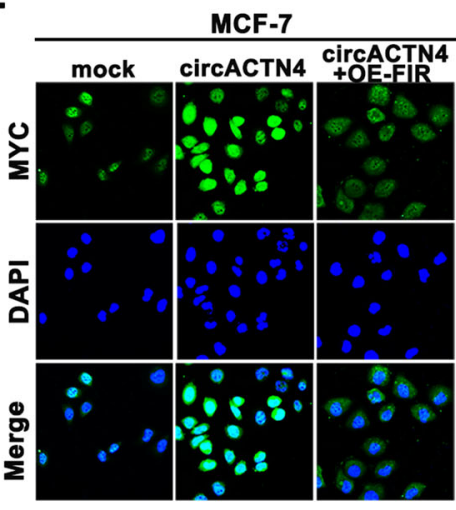

SK-BR-3

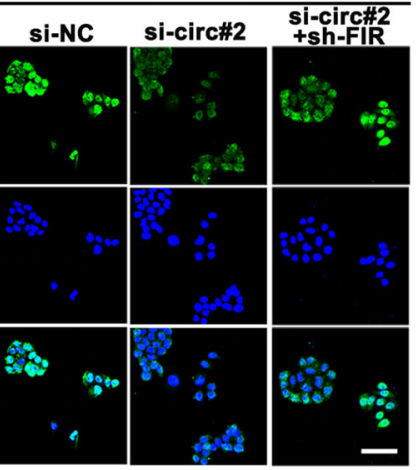

F

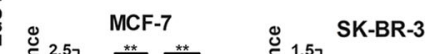

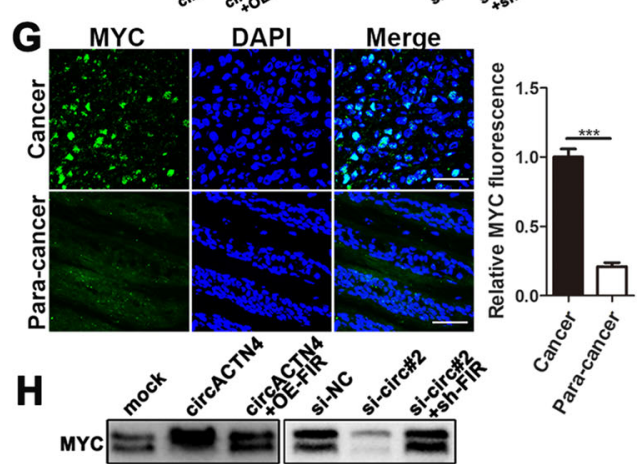
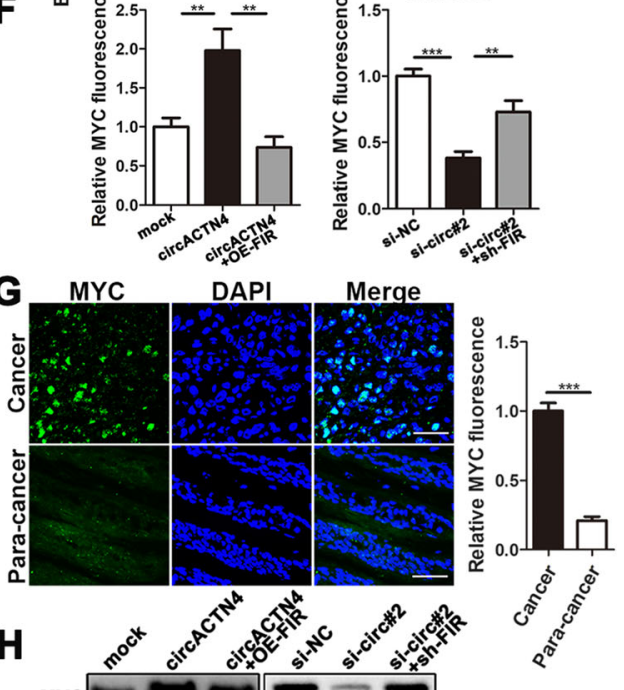

axt

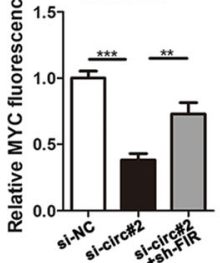

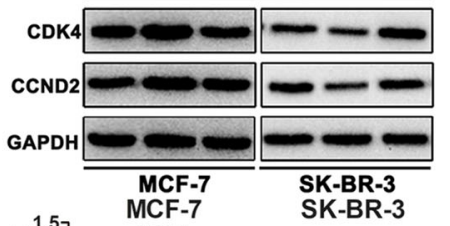

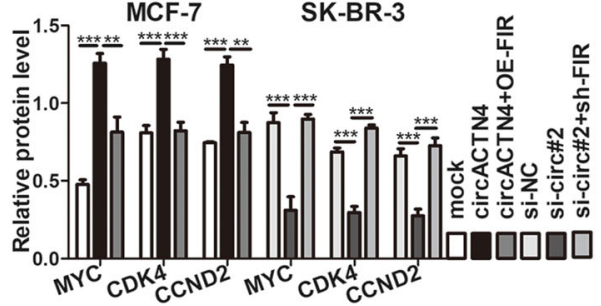

Fig. 7 (See legend on next page.) 
(See figure on previous page.)

Fig. 7 FIR reverses the oncogenic effects of circACTN4 in BC cells. a The ability of cell migration was detected by wound healing assay after transfection or co-transfection with indicated vectors or si-circ\#2 (magnification, $\times 50$, Scale bar, 200 um) . b The ability of cell proliferation was evaluated after transfection and co-transfection with circACTN4 plasmid, si-circACTN4, OE-FIR or sh-FIR vectors by EdU (magnification, $\times 100$, Scale bar, 100 um) assay. $\mathbf{c}$ and $\mathbf{d}$ The invasion and migration abilities of BC cells transfected or co-transfected with circACTN4 plasmid, si-circACTN4, OE-FIR or sh-FIR vectors were measured by transwell invasion and transwell migration (magnification, $\times 100$, Scale bar, 100 um), respectively. e and $\mathbf{f}$ The expression of MYC in BC cells transfected or co-transfected with indicated vectors and siRNA was detected by IF (magnification, $\times 200$, Scale bar, 200 um). $\mathbf{g}$ The expression of MYC in breast cancer and paracancer tissues was displayed by IF (magnification, $\times 400$, Scale bar, 50 um). $\mathbf{h}$ The expressions of MYC and its downstream proteins CDK4 and CCND2 were detected after transfection and co-transfection with indicated vectors and siRNA by western blot. GAPDH was used as the normalizing gene in the above experiments. The data are presented as the mean \pm $\mathrm{SD},{ }^{*} P<0.05,{ }^{* *} P<0.01,{ }^{* * *} P<0.001$

up-regulated or deregulated in up to $70 \%$ of human cancers [38]. For example, it was reported that the level of plasma c-MYC in breast cancer patients was markedly higher than that of normal control and was related to clinical stage and lymph node status [39]. Moreover, overexpression of MYC protein is a characteristic of progression and poor prognosis in multiple myeloma [40]. Overexpressing c-MYC promotes melanoma metastasis and is obviously related with distant metastasis and poor prognosis [41]. Overexpression of lncRNA EPIC1 is associated with poor prognosis in patients with luminal type B breast cancer, and promotes tumor growth and cell cycle progression by interacting with MYC [42]. In present study, we found that circACTN4 could promote the expression of MYC by the binding of FUBP1 to MYC promoter and MYC was highly expressed in BC tissues. MYC as a transcription factor, its main downstream effectors participate ribosome biogenesis, protein translation, metabolism and cell cycle progression [43]. MYC promotes cell-cycle progression through the regulating various genes related to cell cycle. Among the MYC targets, CCND2 forms a complex with CDK4 or CDK6 and functions as a regulatory subunit of the complex, whose activity is crucial for cell cycle G1/S transition [44, 45]. We showed that circACTN4 could promote the expressions of its downstream targets CDK4 and CCND2 by activating the expression of MYC. Our experimental results support previous findings.

Transcription factor upstream stimulatory factor 2 (USF2) plays an important role in tumorigenesis and development. Interestingly, USF2 has been proven to have a dual function as either tumor-suppressor or tumorpromoter [46]. However, the underlying mechanism of tumor inhibition or promotion remains largely unclear. It was reported that USF2 was abnormally high expressed in human breast cancers and correlated with cancer progression [47]. Consistent with these studies, we found that USF2 might bind to ACTN4 gene promoter by bioinformatics analysis, ChIP and luciferase reporter assays. Furthermore, we revealed that upregulation of USF2 could increase the level of
circACTN4, whereas, knockdown of USF2 suppressed the expression of circACTN4, which suggest that USF2 could stimulate ACTN4 gene transcription. Currently, little is known about the regulation mechanisms of upstream transcription factors on circRNA. Our previous research demonstrated that transcription factors E2F1 might promote circSEPT9 gene transcription and enhance circSEPT9 expression [21]. Further research is needed to clarify the exact molecular mechanism for the regulatory process.

\section{Conclusion}

Taken together, we identified a novel circular RNA circACTN4 that was upregulated in human breast cancer tissues and cells, and we found that increased circACTN4 expression was correlated with poor prognosis of breast cancer patients. Furthermore, we demonstrated that overexpression of circACTN4 could effectively increase $\mathrm{BC}$ cell proliferation, invasion and metastasis. Mechanistically, we first discovered that circACTN4 mediated by USF2 might directly bind to FUBP1 and their interaction could impede of the binding of FUBP1 with FIR, thereby activating transcription of MYC and promoting $\mathrm{BC}$ development. Our findings suggest that circACTN4 could be a novel prognostic biomarker and promising therapeutical target for breast cancer.

\section{Supplementary Information}

The online version contains supplementary material available at https://doi. org/10.1186/s12943-021-01383-X.

Additional file 1: Table S1. Primer sequences of $q R T-P C R$ and PCR used in this study. Table S2. Sequences of siRNAs and shRNAs used in this study. Table S3. Sequences of probes used in this study.

Additional file 2: Figure S1. The expression levels of USF2, FUBP1, FIR, ACTN4 and MYC were determined by GRT-PCR or western blot and the binding of FUBP1 and FUSE was detected by CHIP-qPCR. a Relative expression of USF2 in BC cells was evaluated by qRT-PCR after overexpression and knockdown of USF2. $b$ and $c$ The expression of FUBP1 in BC cells was detected by qRT-PCR or western blot after overexpression and knockdown of FUBP1. d and e The expression level of FIR was determined in BC cells after overexpression and knockdown of FIR by qRT-PCR or western blot. $f$ The expression levels of FUBP1 and FIR were detected in BC cells transfected with circACTN4 overexpression plasmids and si- 


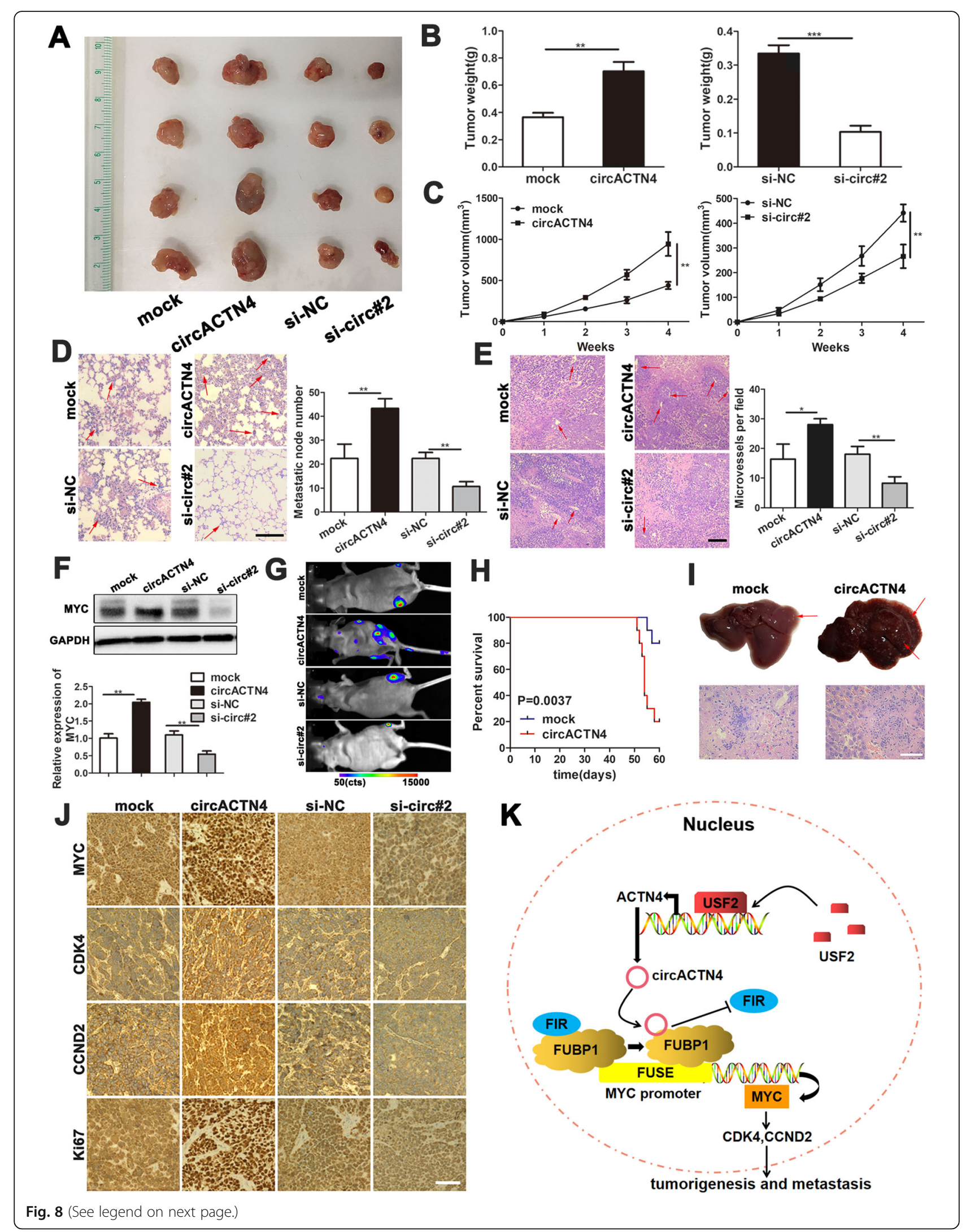


(See figure on previous page.)

Fig. 8 circACTN4 promotes tumorigenesis and metastasis of BC cells. a and $\mathbf{b}$ Representative images of xenograft tumors of each group and tumor weight analysis were shown. $\mathbf{c}$ The tumor volumes were measured once a week and the growth curves were drawn. $\mathbf{d}$ and $\mathbf{e} \mathrm{HE}$ staining of lung and tumor sections were showed. Metastatic nodules of the lungs and microvessels of the tumors were analyzed and indicated with arrows (magnification, $\times 100$, Scale bar, $100 \mu \mathrm{m}$ ). $\mathbf{f}$ Western blot analysis was used to detect the protein level of MYC in xenograft tumor tissues. $\mathbf{g}$ Representative bioluminescent imaging (BLI) of the different groups was shown. circACTN4 knockdown and overexpression MCF-7 cells were injected into tail vein of mice for 4 weeks to detect metastasis ( $n=5$ for each group). $\mathbf{h}$ The survival curve was generated for the nude mice injected with MCF-7 cells infected with overexpressed circACTN4 or mock vector using Kaplan-Meier survival analysis ( $n=10$ for each group). i The representative images of liver metastasis were taken from nude mice injected with circACTN4 overexpressing and mock control MCF-7 cells for 60 days (magnification, $\times 400$, Scale bar, $100 \mu \mathrm{m}$ ). $\mathbf{j}$ The expressions of MYC, CDK4, CCND2 and Ki67 in tumors of nude mice were detected by immunohistochemistry (magnification, $\times 200$, Scale bar, $200 \mu \mathrm{m}$ ). $\mathbf{k}$ The schematic diagram shows how circACTN4 could promote tumorigenesis and metastasis of BC through circACTN4/FUBP1/MYC axis. GAPDH was used as the normalizing gene in the above experiments. The data are presented as the mean $\pm \mathrm{SD},{ }^{*} P<0.05,{ }^{* *} P<0.01,{ }^{* * *} P<0.001$

circ\#2 by western blot. g Relative expression of ACTN4 was determined in 20 pairs BC tissues and para-cancer tissues by qRT-PCR. $h$ Pearson correlation analysis showed that the expression of ACTN4 was positively correlated with the level of circACTN4 in breast cancer tissues. i Relative expression of ACTN4 was evaluated in BC cells after transfection with ACTN4 overexpression or knockdown plasmids by qRT-PCR. $j$ and $k$ The expression level of MYC was detected after transfection or co-transfection with the indicated vectors and siRNA by qRT-PCR and western blot. I Chip assay showed that the transfection of $\mathrm{BC}$ cells with different concentrations of si-circ\#2 had no effect on the binding of FUBP1 and FUSE. GAPD $\mathrm{H}$ was used as the normalizing gene in the above experiments. The data are presented as the mean \pm SD, ${ }^{*} P<0.05,{ }^{* *} P<0.01,{ }^{* * *} P<0.001$.

\section{Acknowledgements}

We thank Department of Endocrine and breast surgery, The First Affiliated Hospital of Chongqing Medical University, Chongqing, China for providing the BC tissue samples and related anonymous clinical data.

\section{Authors' contributions}

JXC designed the research and supervised the project. XSW executed all experiments. LX and RY were responsible for clinical sample collection. HC and MW performed statistical analysis of data. RJ and LYZ provided support experimental technical support. JXC and XSW wrote the manuscript. All authors read and approved the final manuscript.

\section{Funding}

This work was supported by National Natural Science Foundation of China (No. 81672536), Science and Technology Research Program of Chongqing Municipal Education Commission (No. KJZD-K202000405), Innovation research group in Colleges and Universities Program of Chongqing Municipal Education Commission (No. CXQT20012) and the project of the top-notch talent cultivation program for the graduate students of Chongqing Medical University (No. BJRC201918).

\section{Availability of data and materials}

The datasets used and analyzed during the current study are available from the corresponding author on reasonable request.

\section{Declarations}

\section{Ethics approval and consent to participate}

The study was authorized by the Ethics Committee of Chongqing Medical University. All patients signed consent forms. The animal protocols were approved by Chongqing Medical University Animal Care and Use Committee (approval ID: 20180517).

\section{Consent for publication}

All authors give consent for the publication of the manuscript in Molecular Cancer.

\section{Competing interests}

The authors declare that they have no competing interests.

\section{Author details}

${ }^{1}$ Department of Cell Biology and Genetics, Chongqing Medical University, \#1 Yixueyuan Road, Chongqing 400016, China. ${ }^{2}$ Department of Endocrine and breast surgery, The First Affiliated Hospital of Chongqing Medical University, \#1 Yixueyuan Road, Chongqing 400016, China. ${ }^{3}$ Laboratory of Stem Cells and Tissue Engineering, Chongqing Medical University, \#1 Yixueyuan Road, Chongqing 400016, China. ${ }^{4}$ Molecular Medicine and Cancer Research Center, Chongqing Medical University, \#1 Yixueyuan Road, Chongqing 400016, China.

Received: 6 February 2021 Accepted: 31 May 2021

Published online: 11 June 2021

\section{References}

1. Bray F, Ferlay J, Soerjomataram I, Siegel RL, Torre LA, Jemal A. Global cancer statistics 2018: GLOBOCAN estimates of incidence and mortality worldwide for 36 cancers in 185 countries. CA Cancer J Clin. 2018;68(6):394-424. https://doi.org/10.3322/caac.21492.

2. Li J, Sun D, Pu W, Wang J, Peng Y. Circular RNAs in Cancer: Biogenesis, Function, and Clinical Significance. Trends Cancer. 2020;6(4):319-36. https:// doi.org/10.1016/j.trecan.2020.01.012.

3. Liang G, Ling Y, Mehrpour M, Saw PE, Liu Z, Tan W, et al. Autophagyassociated circRNA circCDYL augments autophagy and promotes breast cancer progression. Mol Cancer. 2020;19(1):65. https://doi.org/10.1186/s12 943-020-01152-2.

4. Wang S, Zhang Y, Cai Q, Ma M, Jin LY, Weng M, et al. Circular RNA FOXP1 promotes tumor progression and Warburg effect in gallbladder cancer by regulating PKLR expression. Mol Cancer. 2019;18(1):145. https://doi.org/10.11 86/s12943-019-1078-z

5. Zeng K, Chen X, Xu M, Liu X, Hu X, Xu T, et al. CircHIPK3 promotes colorectal cancer growth and metastasis by sponging miR-7. Cell Death Dis. 2018;9(4):417. https://doi.org/10.1038/s41419-018-0454-8.

6. Wang YG, Wang T, Ding M, Xiang SH, Shi M, Zhai B. hsa_circ_0091570 acts as a ceRNA to suppress hepatocellular cancer progression by sponging hsamiR-1307. Cancer Lett. 2019;460:128-38. https://doi.org/10.1016/j.canlet.201 9.06.007.

7. Qiao J, Liu M, Tian Q, Liu X. Microarray analysis of circRNAs expression profile in gliomas reveals that circ_0037655 could promote glioma progression by regulating miR-214/PI3K signaling. Life Sci. 2020;245:117363. https://doi.org/10.1016/j.lfs.2020.117363.

8. Gao D, Qi X, Zhang X, Fang K, Guo Z, Li L. hsa_circRNA_0006528 as a competing endogenous RNA promotes human breast cancer progression by sponging miR-7-5p and activating the MAPK/ERK signaling pathway. Mol Carcinog. 2019;58(4):554-64. https://doi.org/10.1002/mc.22950.

9. Zou Y, Zheng S, Xiao W, Xie X, Yang A, Gao G, et al. circRAD18 sponges miR-208a/3164 to promote triple-negative breast cancer progression through regulating IGF1 and FGF2 expression. Carcinogenesis. 2019;40(12): 1469-79. https://doi.org/10.1093/carcin/bgz071.

10. Song X, Liang Y, Sang Y, Li Y, Zhang H, Chen B, et al. circHMCU Promotes Proliferation and Metastasis of Breast Cancer by Sponging the let-7 Family. Mol Ther Nucleic Acids. 2020;20:518-33. https://doi.org/10.1016/j.omtn.2020. 03.014.

11. Cao L, Wang M, Dong Y, Xu B, Chen J, Ding Y, et al. Circular RNA circRNF20 promotes breast cancer tumorigenesis and Warburg effect through miR- 
487a/HIF-1alpha/HK2. Cell Death Dis. 2020;1 1:145. https:/doi.org/10.1038/ s41419-020-2336-0.

12. Zang J, Lu D, Xu A. The interaction of circRNAs and RNA binding proteins: An important part of circRNA maintenance and function. J Neurosci Res. 2020;98:87-97. https://doi.org/10.1002/jnr.24356.

13. Huang A, Zheng H, Wu Z, Chen M, Huang Y. Circular RNA-protein interactions: functions, mechanisms, and identification. Theranostics. 2020; 10:3503-17. https://doi.org/10.7150/thno.42174.

14. Lou J, Hao Y, Lin K, Lyu Y, Chen M, Wang H, et al. Circular RNA CDR1as disrupts the p53/MDM2 complex to inhibit Gliomagenesis. Mol Cancer. 2020;19:138. https://doi.org/10.1186/s12943-020-01253-y.

15. Feng $Y$, Yang $Y$, Zhao $X$, Fan $Y$, Zhou L, Rong J, et al. Circular RNA circ0005276 promotes the proliferation and migration of prostate cancer cells by interacting with FUS to transcriptionally activate XIAP. Cell Death Dis. 2019;10:792. https://doi.org/10.1038/s41419-019-2028-9.

16. Dong W, Dai ZH, Liu FC, Guo XG, Ge CM, Ding J, et al. The RNA- binding protein RBM3 promotes cell proliferation in hepatocellular carcinoma by regulating circular RNA SCD-circRNA 2 production. EBioMedicine. 2019;45: 155-67. https://doi.org/10.1016/j.ebiom.2019.06.030.

17. Ni X, Knapp S, Chaikuad A. Comparative structural analyses and nucleotidebinding characterization of the four KH domains of FUBP1. Sci Rep. 2020;10: 13459. https://doi.org/10.1038/s41598-020-69832-z.

18. Debaize L, Troadec MB. The master regulator FUBP1: its emerging role in normal cell function and malignant development. Cell Mol Life Sci. 2019;76: 259-81. https://doi.org/10.1007/s00018-018-2933-6.

19. Liu W, Xiong X, Chen W, Li X, Hua X, Liu Z, et al. High expression of FUSE binding protein 1 in breast cancer stimulates cell proliferation and diminishes drug sensitivity. Int J Oncol. 2020;57:488-99. https://doi.org/10.3892/ijo.2020.5080.

20. Sun Y, Wei G, Luo H, Wu W, Skogerbø G, Luo J, et al. The long noncoding RNA SNHG1 promotes tumor growth through regulating transcription of both local and distal genes. Oncogene. 2017;36:6774-83. https://doi.org/1 0.1038/onc.2017.286

21. Zheng $X$, Huang $M$, Xing $L$, Yang $R$, Wang $X$, Jiang $R$, et al. The circRNA circSEPT9 mediated by E2F1 and EIF4A3 facilitates the carcinogenesis and development of triple-negative breast cancer. Mol Cancer. 2020;19:73. https://doi.org/10.1186/s12943-020-01183-9.

22. Ferlay J, Colombet M, Soerjomataram I, Mathers C, Parkin DM, Piñeros M, et al. Estimating the global cancer incidence and mortality in 2018: GLOBOCAN sources and methods. Int J Cancer. 2019;15(144):1941-53. https://doi.org/10.1002/ijc.31937.

23. Tang Q, Hann SS. Biological Roles and Mechanisms of Circular RNA in Human Cancers. Onco Targets Ther. 2020;13:2067-92. https://doi.org/10.214 7/OTT.S233672.

24. Li H, Yang F, Hu A, Wang $X$, Fang E, Chen $Y$, et al. Therapeutic targeting of circ-CUX1/EWSR1/MAZ axis inhibits glycolysis and neuroblastoma progression. EMBO Mol Med. 2019;1 1:e10835. https://doi.org/10.15252/ emmm.201910835.

25. Yang F, Fang E, Mei H, Chen Y, Li H, Li D, et al. Cis-Acting circ- CTNNB1 Promotes $\beta$-Catenin Signaling and Cancer Progression via DDX3-Mediated Transactivation of YY1. Cancer Res. 2019;79:557-71. https://doi.org/10.1158/ 0008-5472.CAN-18-1559.

26. Ding L, Zhao Y, Dang S, Wang Y, Li X, Yu X, et al. Circular RNA circDONSON facilitates gastric cancer growth and invasion via NURF complex dependent activation of transcription factor SOX4. Mol Cancer. 2019;18:45. https://doi.org/10.1186/s12943-019-1006-2.

27. Quan G, Li J. Circular RNAs: biogenesis, expression and their potential roles in reproduction. J Ovarian Res. 2018;11:9. https://doi.org/10.1186/s13048-01 8-0381-4.

28. Yoshida GJ. Emerging roles of Myc in stem cell biology and novel tumor therapies. J Exp Clin Cancer Res. 2018;37:173. https://doi.org/10.1186/s1304 6-018-0835-y.

29. Zhang J, Chen QM. Far upstream element binding protein 1: a commander of transcription, translation and beyond. Oncogene. 2013;32:2907-16. https://doi.org/10.1038/onc.2012.350.

30. Hsiao HH, Nath A, Lin CY, Folta-Stogniew EJ, Rhoades E, Braddock DT. Quantitative characterization of the interactions among c-myc transcriptional regulators FUSE, FBP, and FIR. Biochemistry. 2010;49:4620-34. https://doi.org/10.1021/bi9021445.

31. Bettegowda C, Agrawal N, Jiao Y, Sausen M, Wood LD, Hruban RH, et al. Mutations in CIC and FUBP1 contribute to human oligodendroglioma. Science. 2011;333:1453-5. https://doi.org/10.1126/science.1210557.
32. Zhang F, Tian Q, Wang Y. Far upstream element-binding protein 1 (FUBP1) is overexpressed in human gastric cancer tissue compared to noncancerous tissue. Onkologie. 2013;36:650-5. https://doi.org/10.1159/0003 55659.

33. Fu PY, Hu B, Ma XL, Tang WG, Yang ZF, Sun HX, et al. Far upstream element-binding protein 1 facilitates hepatocellular carcinoma invasion and metastasis. Carcinogenesis. 2020;41:950-60. https://doi.org/10.1093/carcin/ bgz171.

34. Liu ZH, Hu JL, Liang JZ, Zhou AJ, Li MZ, Yan SM, et al. Far upstream element-binding protein 1 is a prognostic biomarker and romotes nasopharyngeal carcinoma progression. Cell Death Dis. 2015;6:e1920. https://doi.org/10.1038/cddis.2015.258.

35. Hoang VT, Verma D, Godavarthy PS, Llavona P, Steiner M, Gerlach K, et al. The transcriptional regulator FUBP1 influences disease outcome in murine and human myeloid leukemia. Leukemia. 2019:33:1700-12. https://doi.org/1 0.1038/s41375-018-0358-8.

36. Ding Z, Liu X, Liu Y, Zhang J, Huang $X$, Yang $X$, et al. Expression of far upstream element (FUSE) binding protein 1 in human glioma is correlated with c-Myc and cell proliferation. Mol Carcinog. 2015;54:405-15. https://doi. org/10.1002/mc.22114

37. Yang L, Zhu JY, Zhang JG, Bao BJ, Guan CQ, Yang XJ, et al. Far upstream element-binding protein 1 (FUBP1) is a potential c-Myc regulator in esophageal squamous cell carcinoma (ESCC) and its expression promotes ESCC progression. Tumour Biol. 2016;37:4115-26. https://doi.org/10.1007/ s13277-015-4263-8.

38. Dong Y, Tu R, Liu H, Qing G. Regulation of cancer cell metabolism: oncogenic MYC in the driver's seat. Signal Transduct Target Ther. 2020;5: 124. https://doi.org/10.1038/s41392-020-00235-2

39. Shi W, Xu X, Huang R, Yu Q, Zhang P, Xie S, et al. Plasma C-MYC level manifesting as an indicator in progression of breast cancer. Biomark Med. 2019;13:917-29. https://doi.org/10.2217/bmm-2019-0073.

40. Szabo AG, Gang AO, Pedersen M $\varnothing$, Poulsen TS, Klausen TW, Nørgaard P. Overexpression of c-myc is associated with adverse clinical features and worse overall survival in multiple myeloma. Leuk Lymphoma. 2016;57:252634. https://doi.org/10.1080/10428194.2016.1187275.

41. Lin X, Sun R, Zhao X, Zhu D, Zhao X, Gu Q, et al. C-myc overexpression drives melanoma metastasis by promoting vasculogenic mimicry via c-myc/ snail/ Bax signaling. J Mol Med (Berl). 2017;95:53-67. https://doi.org/10.1007/ s00109-016-1452-X.

42. Wang Z, Yang B, Zhang M, Guo W, Wu Z, Wang Y, et al. IncRNA Epigenetic Landscape Analysis Identifies EPIC1 as an Oncogenic IncRNA that Interacts with MYC and Promotes Cell-Cycle Progression in Cancer. Cancer Cell. 2018; 33:706-20.e9. https://doi.org/10.1016/j.ccell.2018.03.006.

43. Chen H, Liu H, Qing G. Targeting oncogenic Myc as a strategy for cancer treatment. Signal Transduct Target Ther. 2018;3:5. https://doi.org/10.1038/ s41392-018-0008-7.

44. Wang C, Yang Y, Zhang G, Li J, Wu X, Ma X, et al. Long noncoding RNA EMS connects c-Myc to cell cycle control and tumorigenesis. Proc Natl Acad Sci U S A. 2019;16(116):14620-9. https://doi.org/10.1073/pnas.1903432116.

45. Hahm ER, Singh KB, Singh SV. c-Myc is a novel target of cell cycle arrest by honokiol in prostate cancer cells. Cell Cycle. 2016;15:2309-20. https://doi. org/10.1080/15384101.2016.1201253.

46. Chi TF, Horbach T, Götz C, Kietzmann T, Dimova EY. Cyclin-Dependent Kinase 5 (CDK5)-Mediated Phosphorylation of Upstream Stimulatory Factor 2 (USF2) Contributes to Carcinogenesis. Cancers (Basel). 2019;11:523. https:// doi.org/10.3390/cancers 11040523.

47. Tan Y, Chen Y, Du M, Peng Z, Xie P. USF2 inhibits the transcriptional activity of Smurf1 and Smurf2 to promote breast cancer tumorigenesis. Cell Signal. 2019;53:49-58. https://doi.org/10.1016/..cellsig.2018.09.013.

\section{Publisher's Note}

Springer Nature remains neutral with regard to jurisdictional claims in published maps and institutional affiliations. 